\title{
nMAT3 is an essential maturase splicing factor required for holo-complex I biogenesis and embryo-development in Arabidopsis thaliana plants
}

Sofia Shevtsov-Tal ${ }^{1}$, Corinne Best ${ }^{1}$, Roei Matan ${ }^{1}$, Sam Aldrin Chandran ${ }^{2}$, Gregory G.

Brown $^{3}$, Oren Ostersetzer-Biran ${ }^{1, *}$

${ }^{1}$ Department of Plant and Environmental Sciences, The Alexander Silberman Institute of Life Sciences, The Hebrew University of Jerusalem, Givat-Ram, Jerusalem 91904, Israel.

${ }^{2}$ School of Chemical and Biotechnology, SASTRA University, Thanjavur 613 401, India.

${ }^{3}$ Department of Biology, McGill University, Montreal, Quebec H3A 1B1, Canada.

* Address correspondence to: Oren Ostersetzer-Biran, PhD, The Hebrew University of Jerusalem, Edmond J. Safra Campus, Givat Ram, Jerusalem, 91904, Israel. Tel. +972-2-6585191; Fax, +972-2-658-5191; E-mail, oren.ostersetzer@mail.huji.ac.il

Running title: "nMAT3-facilitated intron splicing and embryogenesis"

Keywords: maturase / group II intron / splicing / mitochondria / embryogenesis / Arabidopsis 


\section{Summary}

Group II introns are large catalytic RNAs that are particularly prevalent in the organelles of terrestrial plants. In angiosperm mitochondria, group II introns reside in the coding-regions of many critical genes, and their excision is essential for respiratory-mediated functions. Canonical group II introns are self-splicing and mobile genetic elements, consisting of the catalytic intron-RNA and its cognate intron-encoded endonuclease factor (i.e. maturase, PfamPF01348). Plant organellar introns are extremely degenerate, and lack many regions that are critical for splicing, including their related maturase-ORFs. The high degeneracy of plant mitochondrial introns was accompanied during evolution by the acquisition of 'host-acting' protein cofactors. These include several nuclear encoded maturases (nMATs) and various other splicing-cofactors that belong to a diverse set of RNA-binding families, e.g. RNA helicases (Pfam-PF00910), Mitochondrial Transcription Termination Factors (mTERF, Pfam-PF02536), Plant Organelle RNA Recognition (PORR, Pfam-PF11955), and Pentatricopeptide repeat (PPR, Pfam-PF13812) proteins. Previously, we established the roles of MatR and three nuclearmaturases, nMAT1, nMAT2, and nMAT4, in the splicing of different subsets of mitochondrial introns in Arabidopsis. The function of nMAT3 (AT5G04050) was found to be essential during early embryogenesis. Using a modified embryo-rescue method, we show that $n M A T 3$-knockout plants are strongly affected in the splicing of nadl introns i1, i3 and i4 in Arabidopsis mitochondria. The embryo-defect phenotype is tightly associated with complex I biogenesis defects. Functional complementation of $n M A T 3$ restored the splicing defects and altered embryogenesis phenotypes associated with the nmat 3 mutant-line. 


\section{Introduction}

The mitochondrion serves as a hub for cellular energy metabolism in the plant cell (Millar et al. 2011, Schertl and Braun 2014). As descendants from an ancestral proteobacterial symbiont, in the vast majority of species mitochondria contain their own genetic system (mitogenome, mtDNA), which encodes for rRNAs and some of the organellar tRNAs and proteins (Bonen 2018, Gualberto and Newton 2017). Plants are able to coordinate their energy demands during particular growth and developmental stages by means of nucleocytoplasmic signaling (i.e. nuclear to mitochondria or plastids). The metabolic functions, biogenesis and maintenance of the mitochondria are controlled by complex networks of genetic interactions between the host genome and the organelles, activities which may relate to plant terrestrialization about half a billion years ago (Best et al. 2020). For instance, the mitochondrial ribosomes and the energy transduction machineries are assemblies of both nuclear and organellar encoded subunits, where the correct stoichiometry in the accumulation of the different subunits composing the organellar complexes is necessary for their biogenesis and functions. These processes necessitate complex mechanisms for regulating the coordination of the expression and accumulation of the different subunits encoded by the two physically remote genomes (Fuchs et al. 2020, Kleine and Leister 2016, Woodson and Chory 2008). However, the identity of the messenger molecules involved in these pathways is more elusive.

The mtDNAs of land plants are remarkably variable in size, sequence and structure (Bonen 2018, Gualberto and Newton 2017). These may also contain linear or circular extrachromosomal DNAs, and undergo frequent recombination events that lead to rapid changes in genome configuration, and also result in the formation of many novel open reading frames (ORFs), many of which have no assigned functions (Bonen 2018, Gualberto and Newton 2017). Some plants have experienced dramatic expansion in mitogenome size, resulting in the largest known mitochondrial genomes (i.e. $11.3 \mathrm{Mbp}$ ) (Sloan et al. 2012). Others, such as the mitochondrial genome in mistletoe (Viscum album) undergone massive gene loss (Maclean et al. 2018, Petersen et al. 2015, Senkler et al. 2018). Despite the large variation in mitogenome size and gene organization, the number of mitochondrial genes is relatively conserved in the land-plant kingdom, with about 60 known genes found in different terrestrial plant species (Bonen 2018, Grewe et al. 2014, Gualberto and Newton 2017, Guo et al. 2016, Mower et al. 2012, Park et al. 2015, Sloan, et al. 2012). These include tRNAs, rRNAs, ribosomal proteins, various subunits of the respiratory complexes I (NADH dehydrogenase), III (cytochrome c reductase or bc1), and IV (cytochrome c oxidase), subunits of the ATP-synthase (also denoted 
as $\mathrm{CV}$ ), cytochrome $c$ biogenesis (CCM) factors, and at least one component of the twinarginine protein translocation complex.

Different from their counterparts in Animalia, the mitochondrial genes in land plants are arranged in numerous polycistronic units that are physically separated by large intergenic regions, while many of the coding regions contain intron sequences that must be excised posttranscriptionally (Zmudjak and Ostersetzer-Biran 2017). The intergenic regions are postulated to harbor important regulatory regions, such as promoters, enhancer or repressor DNA sequences, which can activate or downregulate the expression of organellar genes in a tissue, developmental or environmental dependent manner. In addition to transcriptional control, posttranscriptional RNA processing seems to play a pivotal role in the regulation of land plant mtDNA expression (Best, et al. 2020, Bonen 2018, Colas des Francs-Small and Small 2014, Hammani and Giege 2014, Zmudjak and Ostersetzer-Biran 2017). These involve the maturation of 5' and 3' termini, RNA editing (typically C-to-U exchanges) (Small et al. 2020) and the removal of intron sequences that reside within many essential protein-coding genes (Brown et al. 2014, Schmitz-Linneweber et al. 2015). These RNA processing steps are essential for the organellar RNAs to carry out their functions in protein synthesis, and may therefore serve as key control points in plant mitochondria gene expression.

The processes that lead to the establishment of functional mRNAs in mitochondria are accomplished largely by nuclear-encoded cofactors, which may also provide a means to link organellar functions with environmental and developmental signals (Best, et al. 2020). Recent data show that the nuclear genomes of angiosperms encode numerous RNA binding proteins essential for mitochondria biology and plant physiology (Colas des Francs-Small and Small 2014, Zmudjak and Ostersetzer-Biran 2017). In fact, these factors comprise a staggering proportion of the total proteome of angiosperm mitochondria, representing about $15 \%$ of the identified protein species (Fuchs, et al. 2020), further signifying the importance of RNA metabolism for mitochondrial biogenesis and plant physiology (Best, et al. 2020).

Our work focuses of group II intron splicing in land plant mitochondria. Although group II introns in plants have evolved from maturase-encoding group II sequences (Ahlert et al. 2006), only the fourth intron in NADH dehydrogenase 1 (nadl i4) in angiosperms has retained an ORF encoding a protein with sequence similarity to maturases (MatR) (Wahleithner et al. 1990). Previously we demonstrated that MatR is associated with nadl i4 and several other intron-containing pre-mRNAs, and facilitates the splicing of the introns with whom it's associated in vivo (Sultan et al. 2016). In addition to MatR, genetic screens have led to the identification of many splicing cofactors in plant mitochondria (see e.g. (Colas des Francs- 
Small and Small 2014). These belong to a diverse set of RNA-binding families, e.g. RNA helicases (Pfam-PF00910), Mitochondrial Transcription Termination Factors (mTERF, PfamPF02536), Plant Organelle RNA Recognition (PORR, Pfam-PF11955), and Pentatricopeptide repeat (PPR, Pfam-PF13812) proteins, while several others are closely related to type-II intron maturases (Pfam-PF01348). For recent reviews about plant mitochondria group II intron splicing see e.g. (Brown, et al. 2014, Colas des Francs-Small and Small 2014, Hammani and Giege 2014, Schmitz-Linneweber, et al. 2015, Zmudjak and Ostersetzer-Biran 2017).

Arabidopsis harbours four maturase-related genes that are found in the nucleus as lone ORFs, with no trace of their corresponding group II intron sequences (Brown, et al. 2014, Mohr and Lambowitz 2003, Schmitz-Linneweber, et al. 2015). Localization analyses indicated that the four nMATs are all localized to the mitochondria, in vivo. Thus, a reasonable hypothesis is that these proteins act in the processing of mitochondrial introns in angiosperms. Previously, we established the roles of nMAT1 (At1g30010) (Keren et al. 2012, Nakagawa and Sakurai 2006), nMAT2 (At5g46920) (Keren et al. 2009, Zmudjak et al. 2017) and nMAT4 (Cohen et al. 2014) in the splicing of different subsets of mitochondrial introns in Arabidopsis.

In this work, we analyzed the RNA profiles and organellar activities associated with nMAT3 (At5g04050), and establish a role for this protein in the splicing of several mitochondrial nadl introns. The effects of lowering the expression of nMAT3, encoded by the At5g04050 gene-locus, on the phenotype and physiology of knockout (T-DNA) rescued lines in Arabidopsis plants are discussed.

\section{Results}

\section{The Arabidopsis $n M A T 3$ (At5g04050) gene-locus encodes a type-II self-standing} maturase factor that is expressed during early developmental stages

Group II introns are both catalytic RNAs and retroelements, which are able to transpose into homologous loci in the host genome, through a process catalyzed by a ribonucleoprotein complex consisting of the released intron and its related maturase ORF (Lambowitz and Zimmerly 2011, Zimmerly and Semper 2015). Typical maturases (MATs) are characterized by two functional domains that are required for both splicing and RNA mobility activities. These include a region with sequence similarity to retroviral-type reverse transcriptases (PfamPF07727), with conserved sequence blocks that are present in the fingers and palm (fingerspalm) regions and a sequence motif similar to the thumb domain of retroviral RTs (also denoted as domain X), which are associated with RNA binding and splicing (Mohr et al. 1993). Some, 
but not all, of the MATs harbor a canonical C-terminal region with homology to DNA-binding endonucleases (i.e. D-En domain) (Pfam-12705).

The nuclear genome of Arabidopsis encodes four maturase-related genes (annotated as nMAT's 1 to 4 ) that their products are postulated to reside in the mitochondria (Brown, et al. 2014, Mohr and Lambowitz 2003, Schmitz-Linneweber, et al. 2015). Figure 1 represents a schematic representation of plant mitochondrial maturase-related proteins. MatR encodes a degenerated MAT protein that harbours some of the finger-palm regions and an intact domain$\mathrm{X}$, but lacks the C-terminal D-En domain (Sultan, et al. 2016). Based on their topology and predicted evolutionary origins the four nMATs in plants are classified as either type-I (i.e. nMAT1 and nMAT2), which harbor the RT and X domains but lack the D-En motifs, and typeII maturases (nMAT3 and nMAT4), that have retained the two domains characteristic of canonical group II intron-encoded maturases (Keren, et al. 2009, Mohr and Lambowitz 2003). While the RT domains in nMAT1 and nMAT2 appear to have degenerated, the D-En regions of nMAT3 and nMAT4 contain amino acid alterations that are expected to inactivate their endonuclease activities (Fig. 1a) (Mohr and Lambowitz 2003). It is therefore expected that the maturases in plants have retained their splicing activities (Cohen, et al. 2014, Keren, et al. 2009, Keren, et al. 2012, Nakagawa and Sakurai 2006, Zmudjak, et al. 2017), but lack the mobility functions associated with the model type-II group II intron-encoded endonucleases.

According to 'The Arabidopsis Information Resource' (TAIR; http://www.arabidopsis.org), the At5g04050 gene-locus (encoding the nMAT3 protein) (Keren, et al. 2009, Mohr and Lambowitz 2003), harbors two intron sequences that are suggested to be alternatively spliced into two isoforms: a 3,027 nucleotide-long unspliced product (At5g04050.1), encoding a 599 amino acids protein (i.e. nMAT3.1), and a spliced (At5g04050.2) mRNA variant of 2,905 nucleotides, encoding a 694 amino acid long protein (nMAT3.2) (Fig. 1b and Supplemental Figure S1). Yet, DNA sequencing of the At5g04050 gene-locus revealed to some errors within the sequence of $n M A T 3$ intron 1 (Fig. S1). BLAST searches indicated that the modified intron sequence is conserved in different Brassicales species, e.g. Arabis alpine, Arabidopsis lyrate, Brassica rapa, Brassica napus, Brassica oleracea, Camelina sativa and Raphanus sativus (Fig. S2). The putative open-reading-frame of the modified At5g04050 gene-locus encodes a 757 amino acids product (instead of 599 aa's) for the unspliced nMAT3.1 isoform. Analyses of nMAT3.1 and nMAT3.2 by the SMART (Letunic et al. 2012) and Conserved Domain Database (CDD) (Marchler-Bauer et al. 2003) servers indicated that the nMAT3.2 variant harbors some of the fingers-palm regions, but lacks the X and D-En domains, whereas the 'unspliced' nMAT3.1 isoform harbors an both the RT 
and D-En motifs, typical to group II intron-encoded maturases (Fig. 1b). The expression of the two putative At5g04050 gene products was further analyzed by RT-PCR, with primers flanking introns 1 and 2. These analyses indicated the existence of only a single isoform, the 'unspliced' nMAT3.1 gene-product in Arabidopsis thaliana (var. Col-0) plants (Fig. S3). Based on these data it is predicted that Arabidopsis harbors only a single functional isoform, the nMAT3.1 gene-product, which encodes an 'intact' maturase-related (nMAT3) protein.

\section{$n M A T 3$ encodes a lowly-expressed protein that is localized to the mitochondria, and have essential roles during early embryo-development}

The TAIR and the 'Genevestigator analysis toolbox' (Hruz et al. 2008, Zimmermann et al. 2004) servers indicate that At5g04050 is a lowly expressed gene, which shows a differential expression throughout development in different organs, with dominant expression in embryonic organs, and young developing tissues, e.g. leaves, apical root meristem, flowers and the shoot apex (Fig. S4). The gene expression databases indicate that nMAT3 expression is mostly upregulated during germination and early plant life (Fig. S4). In accordance with its predicted low-expression levels, nMAT3 could not be found among the different proteins identified in mass-spectrometry analyses of plant organellar fractions, which contain various RNA binding cofactors (i.e. SUBA4 server) (Hooper et al. 2017). nMAT3 is predicted to reside in the mitochondria by the consensus SUBAcon (Hooper, et al. 2017). Previously, we demonstrated by GFP localization analyses that the Arabidopsis $n M A T 3$ gene-locus encodes a mitochondrialocalized protein (Keren, et al. 2009). The homology of At5g04050 gene product(s) with model group II-encoded maturases and its localization by GFP-targeting studies (Keren, et al. 2009) support a role for nMAT3 in the processing of organellar introns in plants.

To study the putative roles of nMAT3 in mitochondria biogenesis, we examined available T-DNA insertional lines at the At5g04050 gene-locus, available at TAIR's seed stock. These include heterozygous SAIL_254_F03 (nmat3-1) line, containing a T-DNA insertion within the RT domain of nMAT3, the homozygous SALK_011307C (nmat3-2) and heterozygous SALK_139392 (nmat3-3) lines that contain an insertion within the putative promoter region of At5g04050 gene, and heterozygous SALK_144082 (nmat3-4) line that contains a T-DNA insertion that is mapped to the 3'-UTR in $n M A T 3$ gene. Sequencing of genomic PCR products, spanning the T-DNA insertional junctions in the four nmat 3 mutants confirmed the integrity of the insertional lines (Fig. 1b, and Fig. S1). No plants homozygous for the $n M A T 3-1$ mutant allele could be recovered among the seeds obtained from heterozygote SAIL_254_F03 line (Fig. 1c). Homozygous nmat3-2, nmat3-3 and nmat3-4 plants (containing 
T-DNA insertions at the 5 ' or $3^{\prime}$ termini of the $n M A T 3$ gene) did not show any obvious phenotypes under normal growth conditions (Keren, et al. 2009). RT-qPCR analysis of nMAT3 expression indicated that the T-DNA insertions did not alter the expression of nMAT3 in the homozygous lines (Keren, et al. 2009).

The selfed progeny of the heterozygous nmat3-1 mutants showed that about a quarter of the seeds did not germinate (i.e. 28 out of 101 seeds), suggesting that $n M A T 3$ encodes an essential gene. The heterozygous nmat3-1 plants were phenotypically indistinguishable from the wild-type plants (Col-0), suggesting that the insertion within the coding region of $n M A T 3$ gene result in a recessive embryo-lethal phenotype, and that a single copy of the gene is sufficient to support normal growth and development. Microscopic analysis of siliques obtained from Arabidopsis wild-type (Col-0; Fig. 2a) and heterozygous nmat3-1 plants (Fig. 2b) showed the presence of translucent seeds in nmat3-1 mutant-line (Fig. 2b, marked with black arrow). These comprised $24.6 \%$ of 693 seeds obtained from 43 siliques of nmat $3-1$ plants, and $0 \%$ white seeds out of 160 seeds obtained from wild-type plants. While seeds were produced normally in Col-0 (Fig. 2c), the mature siliques of nmat $3-1$ contained about $25 \%$ darkened and shrunken seeds (Fig. 2d). Nomarski microscopy analyses further indicated that green seeds carry mature embryos, while the translucent seeds of the same progeny contain embryos arrested at the heart-torpedo transition stage (Fig. 2e-f). During the maturation of the siliques, some of the pale seeds collapsed into seeds lacking embryos (Fig. 2e, indicated by an arrow), while others degenerate into shrunken-brown seeds (Fig. 2e, indicated by an arrow). The morphology of the shrunken seeds resembles that of wrinkledl mutants (Focks and Benning 1998), a seed developmental defect that is also associated with altered mitochondrial activities (Keren, et al. 2012, Kuhn et al. 2015, Pinfield-Wells et al. 2005). While wild-type (Fig. 2g) and 'normal looking' seeds collected from heterozygous nmat3-1 plants germinate successfully on soil or MS-agar plates (Fig. 2g), wrinkled seeds from the same siliques fail to sprout, and did not germinate even when incubated on (1\%) sucrose-containing MS-agar medium (Fig. 2d), a condition used to recover germination-arrested phenotypes in Arabidopsis mutants affected in mitochondria biogenesis, e.g., the codl (Dahan et al. 2014) and call/cal2 mutants (Cordoba et al. 2016, Fromm et al. 2016a).

\section{Embryo rescue of Arabidopsis nMAT3-1 mutant-line}

Arabidopsis mutants impaired in mitochondria biogenesis or functions often display altered seed germination, a phenotype that is associated with embryo developmental defects (Colas des Francs-Small and Small 2014, Cordoba, et al. 2016, Dahan, et al. 2014, Focks and Benning 
1998, Franzmann et al. 1989, Fromm, et al. 2016a, Keren, et al. 2009, Kuhn, et al. 2015, Ostersetzer-Biran 2016, Pinfield-Wells, et al. 2005). Our data show that loss-of-function knockout nMAT3-1 mutant-seeds were not informative for the analysis of nMAT3 functions in Arabidopsis plants. None of the mature wrinkled seeds of nmat3-1, which we considered to be homozygous for the $n M A T 3-1$ allele, were able to germinate. One approach for studying the post-embryonic function of essential genes involves a partial complementation of the mutation, by cloning a target gene under a seed-specific promoter, e.g. ABI3 (Despres et al. 2001) or RPS5A (Johnson et al. 2008) promoters. The technique has been successful in studying various embryo arrested mutants in Arabidopsis (Despres, et al. 2001), among which some are affected in organellar RNA metabolism (Aryamanesh et al. 2017, Sun et al. 2018). However, this method was found unsuitable for the analysis of the nmat3-1 mutant, as no individuals homozygous for the $n M A T 3-1$ allele could be recovered among the progeny of heterozygous nmat3-1 plants carrying the ABI:nMAT3 transgene.

In addition to the use of seed-specific promoters, embryo-rescue approaches have been previously used to recover germination-arrested phenotypes in plant mutants affected in early embryogenesis (Franzmann, et al. 1989), some of which show altered mitochondria biogenesis and function, e.g. codl (Dahan, et al. 2014), ndufvl (Kuhn, et al. 2015) and call/cal2 mutants (Cordoba, et al. 2016, Fromm, et al. 2016a). Unfortunately, the settings described for the rescue of cod1, ndufv1 or call/cal2 mutants, were found insufficient to allow the germination of nmat3-1 seeds, which may relate to the early embryogenesis defect phenotype of nmat3-1 mutant (Fig. 3), whereas the seeds of codl and call/cal2 seem to contain small, but otherwise to fully developed embryos (Cordoba, et al. 2016, Dahan, et al. 2014, Fromm, et al. 2016a).

We tried to overcome this problem, by assaying the germination of nmat3-1 seeds collected from green-matured siliques (i.e. about 10 days post anthesis) (Mizzotti et al. 2018), using various growth media and growth conditions. The optimized settings for germination of homozygous nmat3-1 were obtained when white-translucent seeds, selected from sterilized immature siliques of heterozygous plants, were sown on MS-agar plates supplemented with $1 \%$ sucrose and vitamins (Fig. 3), conditions that we found optimal for the establishment of a PPRrelated (i.e. msp 1025) mutant, which is also arrested at the late heart stage (Best et al. 2019). Under the in vitro conditions, many seeds of wild-type plants containing embryos at the late heart or torpedo stages (Fig. 3a) and a few of the cultivated nmat3-1 seeds germinated after 4 5 weeks (Fig. 3b), whereas a total of about $90 \%$ of green seeds and $40 \%$ of the white seeds germinated during 3 months of incubation. Importantly, genotyping by PCR indicated that seedlings obtained from white-translucent seeds were also homozygous for the loss-of-function 
nMAT3 allele. These slowly developed to the six-true-leaf stage (Boyes et al. 2001) following 2 months of incubation in the growth chamber, but were unable to continue their development beyond the L6-stage (Fig. 3c). When transplanted into a liquid MS-based medium containing $1 \%$ sucrose and vitamins (see Experimental procedures) their development proceeded beyond the L6 stage, and a significant increase in leaf and root biomass was apparent in the culture medium following 4 weeks (Fig. 3d). None of the rescued nmat3 plants were able to complete a life cycle and produce viable seeds. For the sequential analyses of the RNA and protein profiles of nmat3-1 mutants, we used 3 to 4-month-old embryo-rescued plantlets obtained from white seeds obtained from the siliques of heterozygous nmat 3 mutant plants. As control we used embryo-rescued wild-type (Col-0) seedlings, collected from seeds containing embryos at the late heart stage. 3-week-old Arabidopsis MS-grown seedlings were used as a reference for the molecular and biochemical assays used in this study.

\section{Mutations in the $n M A T 3$ gene-locus strongly affect the accumulation or processing of mature nad1 transcripts in Arabidopsis mitochondria}

To gain more insight into the putative roles of nMAT3 in mtRNA metabolism, we used transcriptome analyses of embryo-rescued wild-type and nmat3-1 plants by quantitative reverse transcription PCR (RT-qPCR) (Cohen, et al. 2014, Colas des Francs-Small et al. 2012, Keren, et al. 2012, Sultan, et al. 2016, Zmudjak et al. 2013). The relative accumulation of mitochondrial transcripts (mtRNAs) was estimated by RT-qPCR from the ratios between embryo-rescued nmat3-1 plantlets versus those of 3-week-old MS-agar grown wild-type plants (Fig. 4a). Several genes in Arabidopsis mitochondria are interrupted by group II introns, i.e., ccmFc, cox2, nad1, nad2, nad4, nad5, nad7, rpl2 and rps3 (Unseld et al. 1997). Moreover, several introns have been disrupted by DNA rearrangements, so that separately-transcribed precursors undergo splicing in 'trans'. Accordingly, the maturation of nadl involves the joining of five exons, encoded by three individually-expressed gene fragments (i.e. nad1.1, nad1.2and nad1.3, which are spliced through two trans- (introns 1 and 3) and two cis- (introns 2 and 4) events. Likewise, the maturation of nad 2 involves the splicing of 3 cis-(i.e. nad2 introns 1, 3 and 4) and one trans-spliced intron (nad2 i2) found on two individual transcripts (nad2.1 and nad2.2), whereas the maturation of nad5 involves the joining of five exons, encoded by three individually-expressed gene fragments (i.e. nad5.1, nad5.2 and nad5.1), where introns 2 and 3 are physically separated on the mitogenome and are spliced in 'trans'.

The accumulation of the entire set of splice variants was examined by RT-qPCR with oligos designed to different exon-exon and exon-intron or intron-exon pairs (Table S1). As 
shown in Figure 4a, notable reductions in mtRNA were apparent for different nadl transcripts in nmat3-1 plantlets, where the steady-state levels of transcripts corresponding to nad1 exons a-b (i.e. nadlab) was about 110-fold lower than that of the wild-type plants and about 57,000fold lower in the case of nadlcd transcript, while the accumulation of nadlde transcript was about 80-fold-lower than that of wild-type plants. The expression of other group II introncontaining transcripts, including $c c m F c$, cox2, nad4, nad5, nad7, rpl2 rps3, was not significantly affected in the homozygous nmat3-1 mutant plants. Likewise, no significant effects on the accumulation of mtRNAs corresponding to 'intronless' genes were apparent in the mutant (Fig. 4a). These included cox 1 and cox3 subunits of complex IV, subunits of the ATP synthase complex $(\mathrm{CV})$, cytochrome $\mathrm{C}$ biogenesis and maturation $(\mathrm{ccm})$ factors, and various ribosomal genes, which their mRNAs levels in nmat3-1 were generally comparable to those seen in wild-type plants (Fig. 4a). The roles of nMAT3 in the maturation or processing of nad1 transcripts, was also indicated by northern blot analyses (Fig. S5). Reduced nad1 mRNA was also found for nmat1 mutants affected in the splicing of introns found in nad1, nad2 and nad4 (Keren, et al. 2012, Nakagawa and Sakurai 2006), while reduced nad1, nad4, nad7 and cox2 mRNA levels are found in in nmat2 mutants (Keren, et al. 2009, Zmudjak, et al. 2017) coincide with splicing defects in many of the organellar introns (Fig. S5).

Here, we also analyzed the mtRNA profiles of rescued Col-0 plantlets (Fig. 4a, Col-0 heart), to analyze changes in mtRNA metabolism due to tissue-specific or or developmental effects, as observed during the germination of wheat seeds (Li-Pook-Than et al. 2004). The RNA profile of rescued wild-type embryos at the heart stage was similar to that of the 3-weekold MS-grown wild-type plants, though the steady-state levels of many of the transcripts were slightly upregulated (about $1.5 \mathrm{x} \sim 4.0 \mathrm{x}$ ) in the rescued plantlets (Fig. 4a). No significant changes in the accumulation of mtRNAs was evident in heterozygous nmat3-1 (Fig. 4b). We thus concluded that the observed defects in the expression or processing of nadl transcripts in nmat3-1 relate to RNA metabolism defects and not to developmental effects between embryorescued seedlings and 3-week-old MS-grown plants.

\section{nMAT3 is required for the splicing of nad1 introns 1,3 and 4 in Arabidopsis mitochondria}

The four maturases in Arabidopsis, including the mitochondria-encoded MatR factor (Sultan, et al. 2016), as well as the nuclearly encoded nMAT1 (Keren, et al. 2012, Nakagawa and Sakurai 2006) and nMAT2 (Keren, et al. 2009, Zmudjak, et al. 2017) and nMAT4 (Cohen, et al. 2014) protein, are all function in the splicing of group II introns in Arabidopsis mitochondria. To examine potential effects by nMAT3 on the splicing of nad 1 introns 1,3 and 4, we compared 
the splicing efficiencies (i.e. the ratios of pre-RNAs to mRNAs in mutants versus wild-type plants) of the 23 introns in Arabidopsis mitochondria (Unseld, et al. 1997), between wild-type and nmat3-1 plants (Fig. 5). Splicing defects were determined to be present by the RT-qPCR analyses in cases where the accumulation of a specific pre-RNA was correlated with a reduced level of its corresponding mRNA in the mutant. The analyses indicated strong defects in the maturation of nadl exons a-b, c-d and d-e, where the splicing efficiencies for the splicing of nadl intron 1 (nadl i1), nadl i3 and nadl i4 were reduced by about 7-fold, 19,000-fold and 352-fold, respectively, in comparison to those of wild-type plants (Fig. 5a). Reduced splicing efficiencies in nmat3-1 were also noted for nad2 il (about 5.5-fold) and nad2 i2 (about 2.2fold) (Fig. 5a). The splicing efficiencies of the 23 mitochondrial introns of rescued wild-type embryos (Col-0 heart) and heterozygous nmat3-1 plants were equivalent to those of the 3-weekold MS-gown wild-type plants (Fig. 5b). These data indicate that nMAT3 is pivotal for the trans-splicing of nad1 i3, but is also required for the efficient cis or trans-splicing of nadl introns 1 (trans) and 4 (cis) and nad2 introns 1 (cis) and 2 (trans).

\section{Rescuing nmat3-1 the growth phenotypes and mtRNA metabolism by a constitutive expression of the nMAT3 gene}

To confirm that the observed growth phenotypes and altered organellar RNA metabolism are indeed associated with the loss of nMAT3, we transformed the At5g04050 gene to heterozygous nmat3-1 plants to facilitate a 'functional complementation' of the mutation. For this purpose, the Arabidopsis $n M A T 3$ gene was obtained by PCR, cloned into the shuttle vector pCAMBIA2300 under the control of the constitutive 35S-promoter, and transformed to heterozygous nmat3-1 mutants by the floral-dip method (see Experimental procedure). A single transformant line, which contained the 35S:nMAT3 transgene in the background of a homozygous nmat3-1 mutant-line, was recovered among the progeny of $\mathrm{T} 2$ transformed plants (i.e. nmat3:35S nMAT3). Seeds obtained from the complemented line were germinated on MS-agar medium, and 3-week-old seedlings of nmat3:35S-nMAT3 seedlings were analyzed in regard to their associated organellar RNA profiles. The RT-qPCR analyses indicated that the reduced accumulation of nadl and nad 2 transcripts (Fig. 4b), as well as the splicing defects we see in nad1 introns 1,3 and 4 and nad2 intron 1 and 2 (Fig. 5b) were fully restored in nmat3:35SnMAT3 plants. The genetic data provide with a strong indication that the mutation in $n M A T 3$ gene-locus is directly associated to the mtRNA defects seen in nmat3-1 mutant-line.

\section{Analysis of the biogenesis of organellar respiratory chain complexes in nmat 3 mutants}


The respiratory machinery is made of four major electron transport carriers, denoted as complexes I to IV (i.e. CI, CII, CIII and CIV) and the ATP synthase enzyme (CV), as well as various proteins involved in non-phosphorylating bypasses of the electron transport chain (ETC), i.e. alternative oxidases (AOXs) and rotenone-insensitive $\mathrm{NAD}(\mathrm{P}) \mathrm{H}$ dehydrogenases (NDs) (Jacoby et al. 2012, Millar, et al. 2011, Schertl and Braun 2014, Senkler et al. 2017, Subrahmanian et al. 2016). The altered mtRNA metabolism we saw in nmat3-1 were expected to result in major effects on mitochondrial activities and plant physiology, e.g. (Brown, et al. 2014, Colas des Francs-Small and Small 2014, Zmudjak and Ostersetzer-Biran 2017). NAD1 is essential for CI biogenesis and function (Braun et al. 2014, Fromm et al. 2016b, Ligas et al. 2019, Ostersetzer-Biran 2016). The defects we see in the splicing of nadl introns 1,3 and 4 (Figs. 4 and 5) suggest that NAD1 is absent, or exists in very low levels, in the mitochondria of namt3-1 mutant plants. The steady-state levels of various CI subunits were examined in 3-weekold MS-grown plants, heterozygous nmat3-1 plants, rescued Col-0 and nmat3-1 and complemented nmat3:35S-nMAT3 line by immunoblot analyses with antibodies raised against CA2 ( 30 kDa) (Perales et al. 2005, Sunderhaus et al. 2006), NAD1 ( 36 kDa) and NAD9 ( 23 kDa) (Lamattina et al. 1993). The immunoblot assays indicated that while the steady-state levels of NAD9 and CA2 subunits are equivalent to those of 3-week-old MS-grown wild-type plants, a protein band of $c a$. $50 \mathrm{kDa}$ that corresponds to NAD1 protein was not visible in the nmat3-1 mutant line (Fig. 6a).

The relative abundances of native respiratory complexes I, III, IV and V in 3-week-old wild-type plants, embryo-rescued nmat3-1 and Col-0 seedlings (Col-0 heart), was analyzed by Blue-native (BN) PAGE, followed by 'in-gel' activity assays and immunoblot analyses (Fig. 7). Arrows indicate to the native complexes I ( 1,000 kDa), CIII dimer ( $\left.\mathrm{III}_{2}, \sim 500 \mathrm{kDa}\right)$, the supercomplex $\mathrm{I}^{+} \mathrm{III}_{2}$ (about 1,500 kDa) (Braun, et al. 2014, Senkler, et al. 2017), CIV ( 220 $\mathrm{kDa})$, and $\mathrm{CV}(\sim 600 \mathrm{kDa})$. The BN-PAGE analyses indicated that respiratory chain CI is below detectable levels in homozygous nmat3-1 plants (Fig. 7, and Table S2). No protein bands that correspond to holo-CI (CI) or to supper complexes $\mathrm{I}_{2} \mathrm{III}_{4}$ or I-III 2 (Braun, et al. 2014, Ligas, et al. 2019, Ostersetzer-Biran 2016, Sunderhaus et al. 2010) were observed in BN-gel activity assays and immunoblots of native gels with antibodies raised to the CA2 subunit of the membranous arm or the NAD9 subunit of the soluble arm of CI (Fig. 7). The immunoblots with CA2 subunit CI further indicated the presence of lower molecular weight bands of $c a$. 550, 500, 450 and $200 \mathrm{kDa}$ in nmat3-1 (Fig. 7, labeled as I*). Based on their appearance in the CA2 blot these particles were related to CI assembly intermediates of the membrane arm which are also apparent in various other Arabidopsis mutants affected in CI biogenesis (Braun, et al. 2014, 
Meyer 2012, Meyer et al. 2011, Ostersetzer-Biran 2016). In support of this assumption, the lower mass CI particles are not apparent in in the NAD9 blot (Fig. 7). No significant changes in CI accumulation (i.e., CA2 and NAD9 blots) or activity were observed in the heterozygous nmat3-1 line, nor in BN-PAGE analyses of rescued Col-0 plants (Fig. 7, and Table S2). We noticed that the activities and accumulation of CI were upregulated in embryo-rescued Col-0 plants, as indicated by the in-gel activity assays and immunoblots of CA2 subunit (i.e. 1.3-fold increase) (Fig 7, and Table S2).

BN-PAGE followed by immunoblotting with anti-RISP antibodies indicated to reduced $\mathrm{CIII}_{2}$ levels in nmat3-1 mutant-line (Fig. 7). We also noticed the appearance of a higher molecular particle of $c a .700 \mathrm{kDa}$ in nmat3-1 plants of a yet unknown protein composition. Reduced $\mathrm{CIII}_{2}$ levels and the appearance of the higher molecular mass may relate to altered assembly and biogenesis of CI-CIII 'super-complexes'. Accordingly, the higher mass particle was not apparent in the functionally complemented nmat3:35S nMAT3 line. Notably, the COX native blots also indicated a strong reduction in holo-CIV, which was associated with the appearance of a higher molecular mass particle of $c a .500 \mathrm{kDa}$ containing the COX protein (Fig. 7). The COX2-associated $500 \mathrm{kDa}$ particle was also observed in rescued Col-0 plants, suggesting a tissue or developmental altered CIV assembly, albeit at a lower level. The levels of the $500 \mathrm{kDa}$ particle in $n m a t 3-1$ plants were equivalent to the levels of the holo-CI in rescued Col-0 plants, the heterozygous line and the nmat3:35S nMAT3 complemented line (Fig. 7). As no changes in the expression of organellar genes encoding COX 1-3 subunits of the Cyt.C biogenesis factors, the molecular basis of these effects remains unclear and need to be further investigated. Immunoblots with anti-ATPb antibodies, indicated that $\mathrm{CV}$ accumulates to higher levels in rescued nmat3-1 and Col-0 plants (about 2.1 and 1.8 folds higher, respectively). As the accumulation of holo-CV was not affected in heterozygous nmat3-1 plants or the complemented nmat3:35S nMAT3 line (about 1.03 and 0.97-fold, respectively) (Fig 7, and Table S2), we speculate that CV is likely upregulated during early seedlings development or due to the methodology used for rescuing the nmat3-1 and Col-0 plants.

\section{Arabidopsis nmat3-1 mutants display complex I-specific respiratory defects}

To analyze whether the respiratory activity was affected in nmat3-1 mutant, the oxygen-uptake rates of $100 \mathrm{mg}$ seedlings, obtained from 3-week-old MS-grown wild-type and 3-month-old nmat3-1 mutant plants were monitored in the dark with a Clark-type electrode (Fig. 7). The average $\mathrm{O}_{2}$-uptake rates of nmat3-1 $\left(58.90 \pm 2.62 \mathrm{nmol} \mathrm{O}_{2} \mathrm{~min}^{-1} \mathrm{grFW}^{-1}\right)$ was notably lower than that of wild-type plants $\left(176.48 \pm 12.36 \mathrm{nmol} \mathrm{O}_{2} \mathrm{~min}^{-1} \mathrm{grFW}^{-1}\right)$. The respiratory functions 
in wild-type and nmat 3 mutant were further analyzed in the presence of the electron transport inhibitors rotenone (ROT, $50 \mu \mathrm{M})$, antimycin A (Anti-A, $10 \mu \mathrm{M})$ and potassium cyanide (KCN, $1 \mathrm{mM})$. Pre-incubation with rotenone $(50 \mu \mathrm{M})$, a specific complex I inhibitor, had a strong effect on the respiration rates of wild-type plants (i.e. $51.55 \pm 4.31 \mathrm{nmol} \mathrm{O}_{2} \mathrm{~min}^{-1} \mathrm{gr} \mathrm{FW}^{-1}$ ) (Fig. $8,+\mathrm{ROT}$ ), about $71 \%$ lower than the $\mathrm{O}_{2}$-uptake rates under the standard conditions (Fig. 8, Mock). As also seen in various other mutants affected in CI biogenesis or activity, e.g. (Cohen, et al. 2014, Falcon de Longevialle et al. 2007, Shevtsov et al. 2018), rotenone had a milder effect on the respiratory activities of nmat3 plants (i.e. $46.54 \pm 2.79 \mathrm{nmol} \mathrm{O}_{2} \mathrm{~min}^{-1} \mathrm{grFW}^{-1}$ ), about $21 \%$ inhibition from the control conditions (Fig. 8). Likewise treating the plants with Anti-A, a specific complex III inhibitor, had a marked effect (i.e. about 67\% inhibition) on the respiratory activities of wild-type plants, and a milder effect on the $\mathrm{O}_{2}$-uptake rates of nmat3-1 mutant plants (i.e. about $42 \%$ inhibition) (Fig. 8). The data shown in Figure 8 further indicated that the mitochondrial electron transport inhibition by $\mathrm{CN}^{-}$is more pronounced in $n$ mat $3-1(26 \%$ inhibition), and also had a strong effect on the $\mathrm{O}_{2}$-uptake rates of wild-type plants $(78 \%$ inhibition). These data indicate that CI, but not CII or CIV, was strongly affected in nmat3-1 mutant, and may coincide with the upregulation of alternative pathways of electron transport, via the rotenone-insensitive type II NAD(P)H dehydrogenases (NDs) and the alternative oxidases (AOXs), which can bypass CI and CIV, respectively (see Fig. 6b).

\section{Discussion}

\section{At5g04050 gene-locus encodes an essential mitochondria-localized maturase factor (nMAT3), required for embryo-development in Arabidopsis plants}

The homology of nMAT3 with model maturases (Keren, et al. 2009, Mohr and Lambowitz 2003) and its localization by GFP-targeting studies (Keren, et al. 2009) strongly support a role for nMAT3 in the processing of mitochondrial group II introns in plants. The At5g04050 gene is predicted to encode two putative nMAT3 isoforms: nMAT3.1 that encodes a 757 (i.e. corrected sequence) amino acid product that contains both the RT and D-En domains typical to group II intron-encoded endonucleases (Fig. 1b and Fig. S3), and a spliced variant (nMAT3.2), encoding a 694 amino acid long protein (Fig. 1b and Fig. S3). The postulated product of nMAT3.2 harbors a degenerated RT domain, lacking the highly conserved 'RNA binding and splicing' motif (i.e. the thumb or X domain) of group II maturases, and also lacks the C-terminal D-En domain (Fig. 1b and Fig. S3). Our data support the existence of only a single isoform, the nMAT3.1 gene-product, in Arabidopsis thaliana (var. Col-0) plants (Fig. S3). Gene expression 
databases indicate that At5g04050 is expressed at low levels, with a dominant expression during early developmental stages (Fig. S4).

Macroscopic analyses of siliques obtained from the heterozygous nmat3-1 (SAIL_254_F03) plants indicated a premature arrest of embryo development at the hearttorpedo transition stage (Fig. 2). Here, we sought to define the molecular functions of nMAT3, and to analyze its postulated roles in mtRNA metabolism by establishing loss-of-function mutations in the nMAT3 gene-locus. As no homozygous mutant individuals could be identified among the mature seeds of self-fertilized heterozygous nmat3-1 plants, we used a modified embryo-rescue approach used to recover germination-arrested phenotypes in Arabidopsis (Best, et al. 2019). The in vitro system allowed us to obtain homozygous nmat3-1 plantlets, which displayed severe growth and developmental defect phenotypes (Fig. 2).

\section{nMAT3 is required for processing of pre- nad1 transcripts in Arabidopsis mitochondria}

Comparison between the mtRNA profiles of the homozygous nmat3-1 mutant-line versus Arabidopsis wild-type plant indicated a strong perturbation in the maturation of $\mathrm{nadl}$ exons cd, where the levels of nad1 mRNA was found to be reduced by about 50,000-fold in the mutant (Fig. 4). Reduced levels (i.e. about 100-fold) were also apparent for nadl exons a-b, c-d and de. The accumulation of mature transcripts corresponding to other intron-containing genes, or to various 'intronless' transcripts, was similar to those of the wild-type plants. Analyses of the splicing efficiencies of the 23 introns in Arabidopsis mitochondria (Sloan et al. 2018, Unseld, et al. 1997), indicated a very strong defect in the trans-splicing of nadl i3 in rescued nmat3-1, where the splicing efficiency was reduced by $\sim 20,000$ fold in the mutant-line (Fig. 5). Altered splicing activities were also noted for nadl introns 1 and 4, where their splicing efficiencies in nmat3-1 were found to be about 360 and 20-fold, respectively, lower than those of the wildtype plants (Fig. 5).

We also noted to reduced splicing efficiencies in the cases of nad2 introns 1 and 2 (Fig. 5), but these were significantly less pronounced than those seen for nadl introns 3 and 4 . The mild effects we see in nad2 intron 1 and 2 splicing and the fact that the accumulation of their mRNAs were not affected by the mutation in $n M A T 3$ gene-locus suggest that the maturation of nad2 is indirectly affected by the processing of nad1 introns in Arabidopsis plants, as also previously noted for otp43 (Falcon de Longevialle, et al. 2007), nmat2 (Keren, et al. 2009) (Zmudjak, et al. 2017) and nmat4 (Cohen, et al. 2014) mutants. The mechanisms by which altered expression of particular mt-RNAs influences global mitochondrial gene-expression or RNA metabolism are still under investigation. 
We suggest that the altered mtRNA metabolism in nmat 3 is directly associated with the mutation in At5g04050 gene-locus. This view is strongly supported by the genetic and biochemical analyses. The mtRNA profiles of wild-type embryos rescued at the same developmental stage (i.e. late heart-to-torpedo) were equivalent to those of wild-type, whereas nadl was dramatically reduced in nmat3-1 mutant. Moreover, functional complementation of nmat3-1 restored the organellar RNA and protein profiles associated with the mutation in At5g04050 gene-locus (Figs. 4-7, nmat3:35S-nMAT3 line). Notably, while the mRNA levels of nmat3:35S-nMAT3 were equivalent to those of 3-week-old MS-grown and embryo-rescued wild-type plants (Fig. 4), the splicing efficiencies of many of organellar group II intron were increased in the complemented line (Fig. 5). We speculate that these effects may correspond to off-target effects by nMAT3, which is over-expressed under the $35 \mathrm{~S}$ promoter in nmat 3 mutant (Fig. S6), or might be indirectly associated with the functions of MatR. The organellar MatR factor is encoded within nad 1 i4 of the nad 1.3 transcript, which is strongly upregulated in nmat 3 plants (Fig. 5). MatR was shown to function in the splicing of various group II introns (Sultan, et al. 2016), many of which their splicing is upregulated in the complemented line. It remains possible, however, that altered mtDNA expression caused by nMAT3 over-expression is also influenced by a plethora of metabolic changes and developmental stimuli (Falcon de Longevialle, et al. 2007, Keren, et al. 2012, Zmudjak, et al. 2013).

\section{Mitochondrial complex I biogenesis defects in nmat3 mutants}

Respiratory complex I (NADH:ubiquinone oxidoreductase, EC 7.1.1.2) is an L-shaped enzyme (Baradaran et al. 2013) that is embedded in the inner-mitochondrial membrane, and mediates the transfer of electrons from NADH to ubiquinone. The overall structure of CI has remained conserved from bacteria to animals and plants (Baradaran, et al. 2013). The biogenesis of the 1.0 MDa holo-CI in angiosperms involves the incorporation of $\sim 50$ different subunits, of both mitochondrial (NAD1, NAD2, NAD3, NAD4, NAD4L, NAD5, NAD6, NAD7 and NAD9) and nuclear origin (Lee et al. 2013). These are incorporated into two main assembly intermediates comprising the so-called 'membrane' and 'peripheral' arm-domains (Braun, et al. 2014, Klodmann et al. 2010, Ligas, et al. 2019, Ostersetzer-Biran 2016).

NAD1 is a central anchor component of mitochondrial complex I. In silico and cryoEM analyses indicate that NAD1 is found in the core junction between the two arms of the native complex (Klodmann, et al. 2010, Ostersetzer-Biran 2016, Soufari et al. 2020). Likewise, genetic and biochemical data are indicating that a large reduction in NAD1 expression has a severe consequence on CI assembly and function (Ostersetzer-Biran 2016). The splicing defects 
in $n a d 1$ we see in nmat 3 are expected to influence the biogenesis of holo-CI and the assembly of CI-associated supper complex. Immunoblots of crude organellar preparations indicate that while the CI subunits CA2 and NAD9 are apparent in nmat3-1 mitochondria, NAD1 is below detectable levels in the mutant (Fig. 6). Accordingly, rescued nmat3-1 seedlings show altered respiration and are less sensitive to inhibition by rotenone (Fig. 8), a specific CI inhibitor. BNPAGE revealed that $\mathrm{CI}$ is below detectable levels in the nmat3-1 mutant-line (Fig. 7). The biogenesis defects in CI are likely associated with reduced CI activity (Figs. 7 and 8) and the induction of the alternative electron pathways, as apparent in Figure $6 \mathrm{~b}$.

BN-PAGE followed by immunoblot assays with anti-CA2 antibodies further revealed the formation of partially assembled sub-CI intermediates of the membrane arm, of $c a$. 550, 500, 450 and $200 \mathrm{kDa}$ in nmat3-1 mitochondria (Fig. 7, labeled as I*). These results are in line with various other mutants that are affected in nadl expression, i.e. nmat4 (Cohen, et al. 2014), msp1025 (Best, et al. 2019) or otp43 (Ligas, et al. 2019), which also accumulate CI assembly intermediates that lack complex I activity (Fig. 7). It should be noted, however, that our data are different from those of opt43 and nmat4 mutants that accumulate CI intermediates of $c a$. $200 \mathrm{kDa}$ and $400 \mathrm{kDa}$, but not the $450 \mathrm{kDa}$ or higher mass sub-CI particles (Fig. 7). Yet, similarly to nmat3, the msp1025 mutant also accumulates higher mass sub-CI particles (Best, et al. 2019). The basis for these differences remains unknown, and may correspond to the levels of NAD1 in each mutant line. We speculate that NAD1 in plants might be incorporated into CI later during the membrane-arm assembly, or that CI can be assembled into a large nonfunctional high-molecular weight intermediates that lack NAD1 but contain CA2, as indicated by the immunoblots and in-gel activity assays (Fig. 7).

The signals of $\mathrm{CIII}_{2}$ and CIV were notably reduced in the mutant, and were accompanied by the appearances of higher mass particles that likely correspond to altered CIII and CIV complexes (Fig. 7). As no effects on the expression of organellar genes encoding CIII and CIV subunits, including COX1, COX2, COX3 and the cytochrome c biogenesis (CCM) factors, were observed in nmat3-1 mutant, the molecular basis for these effects in the nmat 3 mutantline remain unclear. It remains possible, however, that these relate to altered nuclear-gene expression, involving CIII and CIV biogenesis related-factors, or represent yet unknown pleiotropic effects that are in particularly ample during early plant development. These speculations await further investigation, although CI defects in plants were previously associated with delayed growth and altered cellular metabolism, as well as various secondary effects on photosynthetic activities (Dutilleul et al. 2003, Meyer et al. 2009, Priault et al. 2006). Various mutants affected in CI biogenesis show defective embryogenesis and low germination 
phenotypes, but are able to be maintained in vitro in media supplemented with vitamins and sugars (Ostersetzer-Biran 2016). The wrinkled morphology of the seeds and their dependency on external sources of energy indicate that seed maturation is incomplete in CI mutants, leading to the production of seeds with reduced reserves and viability (Keren, et al. 2012, Kuhn, et al. 2015). Recent data indicate that once photosynthesis has been established in the young seedlings, growth is no longer dependent on the application of external sugars.

\section{The maturation of nadl is a complex process, involving MatR and the four nuclear- encoded maturases.}

The expression of nadl in vascular plants is a complex process that may represent a key step in the regulation of complex I biogenesis (Ostersetzer-Biran 2016). Analyses of nadl mutants in Chlamydomonas reinhardtii and human mitochondria indicated that NAD1 incorporates at the very earliest stage of complex I assembly (Antonicka et al. 2003, Cardol et al. 2002). However, the incorporation of NAD1 and the molecular pathways that lead to CI assembly in land plants are still under investigation, and may be at variance with this view. At least 12 nuclear-encoded factors (Fig. 9) (Colas des Francs-Small and Small 2014) and one mitochondrial factor (i.e. MatR) (Sultan, et al. 2016), are required for the excision of the four introns within nadl in Arabidopsis mitochondria, and in each case involve at least one maturase protein. The different factors that function in $n a d l$ introns splicing are exemplified in Figure 9. These include the PPR factors MISF74 (Wang et al. 2018) (nadl i4) and OTP43 (Falcon de Longevialle, et al. 2007) (nadl i1), the RNA helicases ABO6 (He et al. 2012) (all four nad1 introns) and PMH2 (Köhler et al. 2010, Zmudjak, et al. 2017) (nadl intron 2 and 3), the CRM-related mCSF1 protein (Zmudjak, et al. 2013) (nadl introns 2 and 3), a RAD52-like protein (Gualberto et al. 2015) ( adl $\mathrm{i} 2$ ), as well as the maturases-associated MatR protein (Sultan, et al. 2016) (nadl introns 3 and 4), nMAT1 (Keren, et al. 2012, Nakagawa and Sakurai 2006) (nad1 i1), nMAT2 (Keren, et al. 2009, Zmudjak, et al. 2017) (nad1 intron 2 and 3), nMAT3 (nad1 introns 1, 3 and 4, this study) and nMAT4 (Cohen, et al. 2014) (nadl introns 1, 3 and 4). Remarkably, nMAT3 and nMAT4, which function in the splicing of the same subset of group II introns in Arabidopsis mitochondria, also seem to be evolutionarily related to one another (Brown, et al. 2014).

The different group II intron splicing factors in Arabidopsis mitochondria are diverse in origin and most likely also in their mechanism of action. PPR proteins may affect intron structures by binding to their RNA targets in a sequence specific manner (Barkan and Small 2014), specify cleavage sites required for the intron maturation and processing. (e.g. (Best, et al. 2019, Lee et al. 2017, Wang et al. 2017), and may also recruit various essential splicing 
factors. Group II maturases and RNA helicases are likely required to stabilize or to nucleate catalytically active structures required for splicing (Brown, et al. 2014, Köhler, et al. 2010, Matsuura et al. 2001, Mohr et al. 2006, Ostersetzer et al. 2005, Schmitz-Linneweber, et al. 2015, Zmudjak, et al. 2017). The extreme complexity of NAD1 expression in Arabidopsis mitochondria further indicates its importance in regulating CI biogenesis in angiosperm species, and may also be associated with the nuclear control of respiratory functions during seed germination and early plant life (Best, et al. 2020). Moreover, the roles of the five mitochondria maturases in Arabidopsis, e.g. MatR, nMAT1, nMAT2, nMAT3 and nMAT4, in the splicing of many introns differ markedly from those of model maturases in bacteria and yeast mitochondria, which influence the processing of their own host introns or closely related RNAs (Schmitz-Linneweber, et al. 2015). This situation, which is also common to other mitochondrial splicing factors in plants, may represent a step in the gradual evolutionary transition from specific maturase-facilitated splicing of self-splicing introns towards the complex spliceosomal machinery in eukaryotes (Schmitz-Linneweber, et al. 2015). Characterization of group II intron splicing in plant mitochondria may also shed light on the functions and mechanisms of their proposed spliceosomal 'descendants' within the nucleus. The homology of the core splicing factor, Prp8, with maturases further supports this idea (Dlakic and Mushegian 2011).

\section{Physiological consequences of respiratory defects in nmat 3 mutants}

Mitochondria have pivotal roles in aerobic cellular metabolism. Altered mitochondrial functions are often associated in plants with embryogenesis defects, reduced germination and growth or developmental-defect phenotypes. Embryo development and the ability to penetrate through the seed coat (Radchuk and Borisjuk 2014) require high amounts of metabolic energy that is provided through the metabolism of macromolecules stored in the seed (Best, et al. 2020). In some plants, as Arabidopsis thaliana, cotyledons absorb much of the endosperm nutrients and thus serve as the main energy supply source for the embryo. Accordingly, embryo development is strongly influenced by the size of the endosperm prior to its breakdown and absorbance by the cotyledons (Fourquin et al. 2016). Roughly on quarter of the seeds in heterozygous lines of nmal, nmat4 and otp43, as well as other mutants affected in mitochondrial gene expression and RNA metabolism are shrunken and brown (Colas des Francs-Small and Small 2014). Similarly, about a quarter of the progeny in the siliques of nmat3-1 were translucent seeds which contain embryos arrested at the heart to torpedo transition stage (Fig. 2). 
Plant embryogenesis and seed-germination are complex developmental processes, which rely on cellular metabolism to support the high-energy demand for the developing embryos. Some Arabidopsis mutants with impaired embryogenesis at the cotyledon stages of development can be rescued on a nutrient medium designed to promote plant regeneration from immature wild-type cotyledons. Mutants arrested at earlier stages in embryogenesis can be also rescued, but these often fail to develop into mature seedlings (Franzmann, et al. 1989). The conditions for the embryo-rescue of nmat 3 mutants involved the addition of vitamins and sugars to promote complete embryo development, and hence seed-germination, and additionally required incubation in liquid medium to support the development beyond the L6 stage (Fig. 3). The growth and development of rescued nmat 3 plantlets was notably inhibited, as compare to rescued wild-type embryos of the same developmental stage (Fig. 3). These were not able to complete a life cycle, nor to flower or set seeds. Why does the loss of nmat 3 cause a major embryo defect, while the seeds of other homozygous mutants affected in nadl processing, e.g., nmat 1, nmat2, nmat 4 or otp 43 mutants, that also produce irregular wrinkled seed are viable? Currently, we cannot provide a definitive explanation to this phenomenon, but we speculate that the variations in growth and development between these mutants relate to the levels of NAD1. While nmat1, nmat4 or otp43 mutants seem to accumulate low levels of nad1 mRNA, the level of NAD1 protein in nmat 3 is below detectable levels (Figs. 4-7). The accumulation of NAD1 in Arabidopsis may therefore relate with embryo maturation during seed development.

In summary, our genetic and biochemical data indicate that nMAT3 is a splicing factor that is pivotal for the expression of NAD1 in Arabidopsis mitochondria, cellular activities that are essential during early embryogenesis in Arabidopsis. Homozygous nmat 3 mutants are strongly affected in the splicing of nadl $i 3$, and also show reduced splicing efficiencies in nadl introns 1 and 4. Consequently, the levels of correctly processed nadl exons c-d RNA (and to a lesser degree also nadl exons a-b and d-e), are substantially reduced. The splicing defects associated with a marked decline in the accumulation of NAD1 protein and severe CI biogenesis defects, where holo-CI is below detectable levels in the nmat3-1 mutant-line. The mtRNA profiles are related to the loss-of-function $n M A T 3-1$ allele, as rescued wild-type embryos at the late heart stage did not show any obvious effects on the accumulation or processing of nadl RNAs. Furthermore, the expression of 35S:nMAT3 in nmat3-1 mutant restored the RNA metabolism defects and altered protein profiles seen in the homozygous line. 


\section{Experimental procedures}

\section{Plant material and growth conditions}

Arabidopsis thaliana (ecotype Columbia) was used in all experiments. Wild-type (Col-0) and nmat3 mutant lines, i.e. SAIL_254_F03 (nmat3-1), SALK_011307C (nmat3-2), SALK_139392 (nmat3-3) and SALK_144082 (nmat3-4) lines were obtained from 'The Arabidopsis Information Resource' (TAIR; http://www.arabidopsis.org) seed stock. Prior to germination, seeds obtained from wild-type and mutant lines were surface-sterilized with $\mathrm{Cl}_{2}$ gas, generated by the addition of $1.5 \mathrm{ml} \mathrm{HCl}$ per $50 \mathrm{~mL}$ of bleach (sodium hypochlorite $4.7 \%$ ), for 4 hours at room-temperature (RT). The seeds were then sown on MS-agar plates containing $1 \%(\mathrm{w} / \mathrm{v})$ sucrose or rescued by a method described in detail below. For synchronized germinations, the seeds were kept in the dark for 5 days at $4^{\circ} \mathrm{C}$ and then grown under long day condition (LD, 16:8-hour) in a controlled temperature and light growth chamber (Percival Scientific, Perry, IA, USA) at $22^{\circ} \mathrm{C}$ and light intensity of $300 \mu \mathrm{E} \mathrm{m}^{-2} \mathrm{~s}^{-1}$. PCR was used to screen the plant collection and check the insertion integrity of each individual line (specific oligonucleotides are listed in Supplemental Table S4). Sequencing of specific PCR products was used to analyze the precise insertion site in the T-DNA lines.

\section{Embryo-rescue and establishment of homozygous nmat3 mutants}

Green-matured siliques (i.e. about 10 days post anthesis), obtained from wild-type and heterozygous nmat3-1 mutant plants, were surfaced sterilized with $6 \%$ bleach solution for 10 min at RT. The seeds were then soaked in a 70\% ethanol solution for $10 \mathrm{~min}$ at RT, washed briefly with sterile DDW, and opened in a biological hood. Green (i.e., wild-type or heterozygous) and white seeds (homozygous), were obtained from siliques of heterozygous nMAT3-1, at different developmental stages (i.e., distributed along the Inflorescence Stem). Likewise, immature seeds of Col-0 seeds at the heart stage were obtained from sterilized siliques. The seeds were then sown on MS-agar plates supplemented with $1 \%(\mathrm{w} / \mathrm{v})$ sucrose and different vitamins (i.e., Myoinositol, Thiamine, Pyridoxine, and nicotinic acid). For DNA and RNA analysis we used Arabidopsis wild-type and nMAT3 plantlets at stage L6 (i.e., 6 to 8 leaves) (Boyes, et al. 2001). To obtain larger quantities of plant material, plantlets at stage L6 grown on MS-agar plates, were transplanted into a MS-based liquid medium supplemented with 1 to $3 \%(\mathrm{w} / \mathrm{v})$ sucrose and vitamins (as above) and incubated in Arabidopsis growth chambers (Percival Scientific, Perry, IA, USA), under standard growth conditions with moderate (50 100 RPM) shaking (i.e., long day conditions, at $22^{\circ} \mathrm{C}$ and light intensity of $300 \mu \mathrm{E} \mathrm{m}^{-2} \mathrm{~s}^{-1}$ ). 


\section{Plant transformation and functional complementation of the nmat3-1 mutation}

nMAT3 clone was generated by subcloning of a 2,425 bp fragment containing the full coding region (including the "introns") of $n M A T 3$ gene under the constitutive cauliflower mosaic virus $35 \mathrm{~S}$ promoter (35S) or the seed-specific $A B I 3$ promoter (Despres, et al. 2001). The construct was cloned into pCAMBIA-2300 (Addgene). The shuttle vector containing the transgene was electroporated into Agrobacterium tumefaciens (strain GV3101), that was used to transform heterozygote nmat3-1 plants by the floral dip method (Clough and Bent 1998). Seeds collected from individual T1 plants, were surface sterilized, and plated on MS-agar medium supplemented with kanamycin $(50 \mu \mathrm{g} / \mathrm{ml})$. Kanamycin-resistant $\mathrm{T} 2$ seedlings were transferred to the soil, grown to maturity and screened for the presence of the transgene (in the genetic background of homozygous nmat3-1). Seeds collected from positive plants were surface sterilized, plated on MS-agar medium supplemented with Kanamycin $(50 \mu \mathrm{g} / \mathrm{ml})$, and then analyzed for their RNA and protein profiles, organellar activities and plant phenotypes.

\section{Microscopic analyses of Arabidopsis wild-type and mutant plants}

Analysis of whole plant morphology, roots, leaves, siliques and seeds of wild-type and mutant lines were examined under a Stereoscopic (dissecting) microscope or light microscope at the Bio-Imaging unit of the Institute of Life Sciences (The Hebrew University of Jerusalem).

\section{RNA extraction and analysis}

RNA extraction and analysis were performed essentially as described previously (Cohen, et al. 2014, Keren et al. 2011, Shevtsov, et al. 2018, Sultan, et al. 2016, Zmudjak, et al. 2013). RNA was prepared from $50 \mathrm{mg}$ seedlings grown on MS-agar plates supplemented with $1 \%$ sucrose, following standard TRIzol Reagent protocols (Ambion, Thermo Fisher Scientific) with additional phenol/chloroform extraction procedure. The RNA was treated with DNase I (RNase-free) (Ambion, Thermo Fisher Scientific) prior to its use in the assays. RT-qPCR was performed with specific oligonucleotides designed to amplify exon-exon (mRNAs) regions, corresponding to numerous mitochondrial genes, and intron-exon regions (pre-mRNAs) within each of the 23 group II introns in Arabidopsis thaliana (Table S1). Reverse transcription was carried out with the Superscript III reverse transcriptase (Invitrogen), using 3 - $5 \mu \mathrm{g}$ of total RNA and $250 \mathrm{ng}$ of a mixture of random hexanucleotides (Promega) and incubated for $50 \mathrm{~min}$ at $50^{\circ} \mathrm{C}$. Reactions were stopped by $15 \mathrm{~min}$ incubation at $70^{\circ} \mathrm{C}$ and the RT samples served directly for real-time PCR. Quantitative PCR (qPCR) reactions were run on a LightCycler 480 
(Roche), using $2.5 \mu \mathrm{L}$ of LightCycler $480 \mathrm{SYBR}$ Green I Master mix and $2.5 \mu \mathrm{M}$ forward and reverse primers in a final volume of $5 \mu \mathrm{L}$. Reactions were performed in triplicate in the following conditions: pre-heating at $95^{\circ} \mathrm{C}$ for $10 \mathrm{~min}$, followed by 40 cycles of $10 \mathrm{sec}$ at $95^{\circ} \mathrm{C}$, $10 \mathrm{sec}$ at $58^{\circ} \mathrm{C}$ and $10 \mathrm{sec}$ at $72^{\circ} \mathrm{C}$. GAPDH (AT1G13440), ACTIN2 (At3g1878), $18 S$-rRNA (At3g41768), RRN26 (Atmg00020), RRN5 (Atmg01380) and RRN18 (Atmg01390), were used as reference genes in the qPCR analyses.

\section{Crude mitochondria preparation from MS-grown Arabidopsis seedlings}

Crude mitochondria extracts were prepared essentially as described previously (Pineau et al. 2008, Shevtsov, et al. 2018). For the preparation of organellar extracts, about $200 \mathrm{mg}$ of liquid MS-grown plantlets were harvested and homogenized in $2 \mathrm{ml}$ of $75 \mathrm{mM}$ MOPS-KOH, pH 7.6, 0.6 M sucrose, $4 \mathrm{mM}$ EDTA, $0.2 \%$ polyvinylpyrrolidone-40, $8 \mathrm{mM}$ L-cysteine, $0.2 \%$ bovine serum albumin and protease inhibitor cocktail ‘complete Mini' from Roche Diagnostics GmbH (Mannheim, Germany). The lysate was filtrated through one layer of miracloth and centrifuged at $1,300 \times \mathrm{g}$ for $4 \mathrm{~min}$ at $4^{\circ} \mathrm{C}$ (to remove cell debris). The supernatant was then centrifuged at $22,000 \times \mathrm{g}$ for $10 \mathrm{~min}$ at $4^{\circ} \mathrm{C}$. The resultant pellet, which contains thylakoid and mitochondrial membranes, was washed twice with $1 \mathrm{ml}$ of wash buffer $37.5 \mathrm{mM}$ MOPS-KOH, 0.3 M sucrose and 2mM EDTA, pH 7.6.

\section{Protein extraction and analysis}

An aliquot equivalent to $10 \mathrm{mg}$ crude mitochondria extract or total protein extract were mixed with an equal volume of $3 X$ protein sample buffer (Laemmli 1970), supplemented with $50 \mathrm{mM}$ $\beta$-mercaptoethanol, and subjected to $12 \%$ SDS-PAGE (at a constant $100 \mathrm{~V}$ ). Following electrophoresis, the proteins were transferred to a PVDF membrane (BioRad) and blotted overnight at $4{ }^{\circ} \mathrm{C}$ with specific primary antibodies (Table S3). Detection was carried out by chemiluminescence assays after incubation with an appropriate horseradish peroxidase (HRP)conjugated secondary antibody (Sigma or Santa Cruz).

\section{Blue native (BN) electrophoresis for isolation of native organellar complexes}

Blue native (BN)-PAGE of crude organellar membranous fractions was performed generally according to the method described by Pineau, et al. (2008). An aliquot equivalent to $40 \mathrm{mg}$ of crude Arabidopsis mitochondria extracts, obtained from wild-type and nMAT3 plants was solubilized with 5\% (w/v) digitonin in BN-solubilization buffer (30 mM HEPES, pH 7.4, 150 $\mathrm{mM}$ potassium acetate, $10 \%[\mathrm{v} / \mathrm{v}]$ glycerol), and then incubated on ice for $30 \mathrm{~min}$. The samples 
were centrifuged $8 \mathrm{~min}$ at $20,000 \mathrm{xg}$ to pellet any insoluble material and Serva Blue $\mathrm{G}(0.2 \%$ $[\mathrm{v} / \mathrm{v}]$ ) was added to the supernatant. The samples were then loaded onto a native 4 to $16 \%$ linear gradient gel. For 'non-denaturing-PAGE' immunoblotting, the gel was transferred to a PVDF membrane (Bio-Rad) in Cathode buffer (50 mM Tricine and $15 \mathrm{mM}$ Bis-Tris-HCl, pH 7.0) for $16 \mathrm{~h}$ at $4^{\circ} \mathrm{C}$ at constant current of $40 \mathrm{~mA}$. The blots where then incubated with antibodies (Table S3) against various organellar proteins, and detection was carried out by chemiluminescence assay with an appropriate horseradish peroxidase (HRP)-conjugated secondary antibody. For CI in-gel activity the gels were washed several times with DDW and the activity assays were performed essentially as described by Meyer, et al. (2009).

\section{Respiration activity}

Oxygen consumption (i.e. $\mathrm{O}_{2}$ uptake) measurements were performed with a Clarke-type oxygen electrode, and the data feed was collected by Oxygraph-Plus version 1.01 software (Hansatech Instruments), as described previously (Shevtsov, et al. 2018). The electrode was calibrated with oxygen-saturated water and by the addition of excess sodium dithionite for complete depletion of the oxygen in water housed in the electrode chamber. Equal weights (200 mg) of wild-type and NMAT3 seedlings were immersed in water and incubated in the dark for a period of $30 \mathrm{~min}$. Total respiration was measured at $25^{\circ} \mathrm{C}$ in the dark following the addition of the seedlings to $2.5 \mathrm{~mL}$ of sterilized tap water in the presence or absence of rotenone $(50 \mu \mathrm{M})$, Antimycin A $(10 \mu \mathrm{M})$ and $\mathrm{KCN}(1 \mathrm{mM})$.

\section{Acknowledgments}

We thank Dr. Michal Zmudjak for her help with establishing homozygous nmat3 plants, and Nadav Biran-Ostersetzer for his help with figure preparation. We also wish to thank Dr. Etienne Meyer (Halle U.) for providing us with anti-NAD1 antibodies. The authors confirm that they have no conflict of interest to declare. This work was supported by grants to O.O.B from the 'Israeli Science Foundation' ISF grants no. 741/15 and 1834/20. 


\section{Figure legends}

Figure 1. Schematic representation of the $n M A T 3$ (At5g04050) gene.

(a) Schematic representation of plant mitochondrial maturase-related proteins. The figure is modified from (Sultan, et al. 2016). Shaded boxes represent different motifs associated with model group II intron-encoded endonucleases. These include the reverse transcriptase (RT) domain (Pfam-PF01348), with its intrinsic NTD (N-terminal domain), the finger-palm (RT I to VII motifs), and the RNA-binding and splicing subdomains (i.e. domain X or thumb; Pfam08388). Some members of this family also harbor an additional C-terminal DNA binding and endonuclease domain (D-En, Pfam-12705). (b) Schematic representation of the nMAT3 gene and its two related gene-products, nMAT3.1 and nMAT3.2. The different motifs are highlighted within the sequence of nMAT4, according to Mohr and Lambowitz (2003) and Sultan, et al. (2016). The location of the T-DNA insertion sites is indicated. (c) PCR-based genotyping of the progeny from heterozygous nmat3 (SAIL_254_F03) plants. Primers are listed in Table S4.

\section{Figure 2. Plant phenotypes associated with nmat3 mutations.}

Mature siliques were obtained from Arabidopsis wild-type (Col-0) and heterozygous nmat3-1 plants. The effects of loss of nMAT3 on seed development and morphology (a-f), and seed germination (g-i) of Arabidopsis wild-type (Col-0) (panels a, c, and g) and knockout nmat3-1 mutant line (b-f). Embryo-developmental phenotypes in green and white seeds of the same progeny of nmat3-1 plants. Images taken with differential interference contrast (i.e. Nomarski) microscopy. Bars in panels $\mathrm{c}$ and represent $1 \mathrm{~mm}$ and $0.1 \mathrm{~mm}$ in panels e and $\mathrm{f}$.

\section{Figure 3. Plant phenotypes associated with rescued homozygous nmat3-1 mutant line.}

Seeds were collected under sterile conditions from surface sterilized immature siliques of Col0 and heterozygous nmat $3-1$ plants. While the seeds of wild-type plants show normal development, the siliques of nmat3-1 mutants contain green (i.e. wild-type or heterozygous embryos), white (homozygous nmat3 embryos) and aborted (degenerated embryos) nmat3-1 mutant seeds (dark yellow). Pictures represent embryo rescue of (a) wild-type seeds at the heart stage and (b) white seeds collected from the immature siliques of heterozygous nmat3-1 plants, following 27 days of incubation on MS-agar plates supplemented with vitamins and sugars (see Experimental procedures). (c) A 2-month-old rescued homozygous nmat3-1 seedling at the L6 stage. (d) 3-month-old rescued wild-type plants transferred to liquid MS-media supplemented 
with $1 \%$ sucrose and vitamins (see Experimental procedure). (e) Image of 3-month-old rescued nmat3-1 plantlet in the liquid MS-media.

\section{Figure 4. Abundance of mitochondrial transcripts in the nmat3-1 mutant.}

Transcriptome analyses of mitochondrial mRNAs levels in Arabidopsis plants by RT-qPCR was performed essentially as described previously (Cohen, et al. 2014, Sultan, et al. 2016, Zmudjak, et al. 2013). RNA extracted from 3-week-old seedlings of wild-type plants, 4 5 months old rescued nmat3-1 mutant plants and plantlets obtained from the immature seeds of Col-0 plants containing embryos at the heart stage (Fig. 3), was reverse-transcribed, and the relative steady-state levels of cDNAs corresponding to the different organellar transcripts were evaluated by qPCR with primers which specifically amplified mRNAs (Supplemental Table S1a). Histograms showing the relative mRNAs levels (i.e. $\log 2$ ratios) in (a) rescued homozygous nmat3-1 mutant and rescued Col-0 plantlets, or (b) heterozygous nmat3-1 mutants and nmat3:35S-nMAT3 complemented plants, versus those of 3-week-old MS-grown wild-type plants. Transcripts analyzed in these assays include the NADH dehydrogenase (CI) subunits nad1 exons a-b, b-c, c-d, d-e, nad2 exons a-b, b-c, c-d, d-e, nad3, nad4 exons a-b, b-c, c-d, nad4L, nad5 exons a-b, b-c, c-d, d-e, nad6 subunit, nad7 exons a-b, b-c, c-d, d-e, and nad9, the complex III cob subunit, cytochrome oxidase (complex IV) cox1, cox2 exons a-b and cox3 subunits, the ATP synthase (i.e., complex V) subunits atp1, atp4, atp6, atp8 and atp9, genes encoding the cytochrome c maturation $(\mathrm{ccm})$ factors, $c \mathrm{cmB}, \mathrm{ccm} C, \mathrm{ccmFn} 1, \mathrm{ccmFn} 2$ and $\mathrm{ccmFc}$ exons a-b, the ribosomal subunits $r p l 2$ exons a-b, rps3 exons a-b, rps4, rps 7 , rps 12, rpl16, $r r n 26$, and the $m t t B$ gene. The values are means of nine RT-qPCR reactions corresponding to three biological replicates (error bars indicate one standard deviation), after normalization to the GAPDH (AT1G13440), ACTIN2 (At3g1878), 18S-rRNA (At3g41768), RRN26 (i.e. mitochondrial 26S-rRNA, Atmg00020), RRN5 (Atmg01380) and RRN18 (Atmg01390) genes.

\section{Figure 5. Splicing efficiencies in nmat3-1 mutants.}

The relative accumulation of mRNA and pre-RNA transcripts in wild-type and rescued homozygous namt $3-1$ plants, corresponding to the 23 group II intron sequences in Arabidopsis, was evaluated by RT-qPCR, essentially as described previously (Cohen, et al. 2014, Sultan, et al. 2016). RNA extracted from wild-type (Col-0), embryo-rescued nmat3 mutants or Col-0 plants at the heart stage (Fig. 3) and nmat3:35S-nMAT3 complemented plants was reversetranscribed, and the relative steady-state levels of cDNAs corresponding to the different organellar transcripts were evaluated by qPCR with primers which specifically amplified pre- 
RNAs and mRNAs (Supplemental Table S1b). The histograms show the splicing efficiencies as indicated by the $\log 2$ ratios of pre-RNA to mRNA transcript abundance in (a) rescued homozygous nmat3-1 mutant and rescued Col-0 plantlets, or (b) heterozygous nmat3-1 mutants and nmat3:35S-nMAT3 complemented plants, versus those of 3-week-old MS-grown wild-type plants. The values are means of 15 RT-qPCR reactions corresponding to five biological replicates (error bars indicate one standard deviation), after normalization to the GAPDH (AT1G13440), ACTIN2 (At3g1878), 18S-rRNA (At3g41768), RRN26 (i.e. mitochondrial 26SrRNA, Atmg00020), RRN5 (Atmg01380) and RRN18 (Atmg01390) genes.

\section{Figure 6. Relative accumulation of organellar proteins in wild-type and nmat3-1 plants.}

(a) Immunoblots with total proteins (about $50 \mu \mathrm{g}$ ), extracted from 3-week-old MS-grown wildtype (Col-0) plants, rescued Col-0 (heart) or nmat3-1 plants, and complemented nmat3-1:35SnMAT3 mutants. The blots were probed with polyclonal antibodies raised to NADHoxidoreductase subunit 1 (NAD1), $\gamma$-carbonic anhydrase-like subunit 2 (CA2) and NAD9 proteins. (b) Analyses of the expression levels of different alternative oxidases (AOXs) and rotenone-insensitive $\mathrm{NAD}(\mathrm{P}) \mathrm{H}$ dehydrogenases (NDs) in Arabidopsis plants was performed by RT-qPCR, essentially as described previously (Cohen, et al. 2014, Sultan, et al. 2016, Zmudjak, et al. 2013). RNA extracted from wild-type (Col-0) and embryo-rescued nmat3-1 mutant plants, was reverse-transcribed, and the relative steady-state levels of cDNAs corresponding to the different $A O X$ and $N D$ transcripts were evaluated by qPCR. The histogram shows the relative mRNAs levels (i.e. log2 ratios) in rescued nmat3 mutant line and Col-0 plantlets, versus 3week-old MS-grown wild-type plants.

\section{Figure 7. Relative accumulation of native organellar complexes in nmat3-1 mutants.}

Blue native (BN)-PAGE analyses of crude organellar membranous fractions were generally performed according to the method described by Pineau, et al. (2008) and Sultan, et al. (2016). An aliquot equivalent to $40 \mathrm{mg}$ of crude Arabidopsis mitochondria extracts, obtained from wildtype (Col-0), rescued Col-0 plants (heart) or nmat3-1 plants, and complemented nmat3-1:35S$n M A T 3$, was solubilized with $5 \%(\mathrm{w} / \mathrm{v})$ digitonin, and the organellar complexes were resolved by BN-PAGE. For immunodetection, the proteins were transferred from the native gels onto a PVDF membrane. The membranes probed with antibodies raised to complex I (CI) NADHoxidoreductase subunit 9 (NAD9) and $\gamma$-carbonic anhydrase-like subunit 2 (CA2) proteins, Rieske iron-sulfur protein (RISP) of complex III (CIII), the cytochrome oxidase subunit 2 
(COX2) of complex IV (CIV), mitochondrial ATPb subunit of ATP-synthase (CV) (Supplemental Table S3), as indicated in each blot. In-gel complex I activity assays were performed essentially as described previously (Meyer, et al. 2009). Arrows indicate to the native supercomplex I+III2 $(\sim 1,500 \mathrm{kDa})$, holo-CI $(\sim 1,000 \mathrm{kDa}), \mathrm{III}_{2}(\sim 500 \mathrm{kDa}), \mathrm{CIV}(\sim 220$ $\mathrm{kDa})$ and $\mathrm{CV}(\sim 600 \mathrm{kDa})$, while $\mathrm{I}^{*}$ indicates the presence of $\mathrm{CI}$ assembly intermediates.

\section{Figure 8. Respiration activity in wild-type and $n M A T 3-$ reduced lines.}

The $\mathrm{O}_{2}$-uptake rates of wild-type (Col-0) and embryo-rescued homozygous nmat3-1 plants, were analyzed with a Clark-type electrode as described previously (Cohen, et al. 2014, Zmudjak, et al. 2017). For each assay, $100 \mathrm{mg}$ MS-grown seedlings were submerged in $2.5 \mathrm{~mL}$ sterilized water and applied to the electrode in a sealed glass chamber in the dark. $\mathrm{O}_{2}$-uptake rates were measured in the absence (Mock) or presence of rotenone (ROT, $50 \mu \mathrm{M}$ ), Antimycin A (Anti-A, $10 \mu \mathrm{M})$ and $\mathrm{KCN}(1 \mathrm{mM})$ that inhibit complexes I, III and IV activities, respectively. The values are means of three biological replicates (error bars indicate one standard deviation), while asterisks indicate significant differences from wild-type plants (Student's T-test, P 0.05).

\section{Figure 9. Target nad1 introns and their accessory splicing cofactors.}

The maturation of nadl in Arabidopsis mitochondria involves the joining of five exons, encoded as three distinct transcription units, one spanning exon nadla (i.e. nad1.1), a second spanning exons nadlb and nadlc (nad1.1) and a third spanning exons nadld and nadle (nad1.1), which are spliced through two trans- (introns 1 and 3) and two cis- (introns 2 and 4) events. The figure shows a schematic representation of $n a d l$ with exons (Ex) depicted as boxes and introns as lines. Specific gene loci are indicated according to the de-novo assembly of the Arabidopsis Col-0 mitogenome (BK010421) (Sloan, et al. 2018). The trans-spliced introns 1 and 3 of $n a d l$ are indicated as an interrupted dashed line. The matR reading frame is shown as a dark box within nadl intron 4. The figure represents various factors that have been demonstrated genetically to be involved for splicing of nadl introns. These include the PPR factors MISF74 (Wang, et al. 2018) and OTP43 (Falcon de Longevialle, et al. 2007), RNA helicases ABO6 (He, et al. 2012) and PMH2 (Köhler, et al. 2010, Zmudjak, et al. 2017), the CRM-related mCSF1 protein (Zmudjak, et al. 2013), the RAD52-like protein (Gualberto, et al. 2015), and the maturases MatR (Sultan, et al. 2016), nMAT1 (Keren, et al. 2012, Nakagawa and Sakurai 2006), nMAT2 (Keren, et al. 2009, Zmudjak, et al. 2017), nMAT3 (indicated by box) and nMAT4 (Cohen, et al. 2014). In addition to these factors, various PPR proteins were shown to determine cleavage sites required for the maturation and processing of group II 
introns, e.g. (Best, et al. 2019, Lee, et al. 2017, Wang, et al. 2017), or may recruit various essential splicing factors, as maturases and RNA helicases which are likely required to stabilize or to nucleate catalytically active structures required for splicing

\section{Supplemental Materials}

Supplemental Figure S1. The nucleotide sequence of $n M A T 3$ gene and the locations of nmat3-1 T-DNA-insertional line.

The nucleotide sequence of $n M A T 3$, encoded by At5g04050 gene-locus. Underlined letters indicate to the 5' and 3' untranslated regions (UTRs), as indicated by the RACE analysis and TAIR database, while uppercase letters represent the open reading frame of nMAT3. The position of T-DNA insertion in nmat3-1 (SAIL_254_F03) line is indicated within the sequence of $n M A T 3$. The location of the T-DNA insertion within nMAT3 gene was analyzed by PCR and sequencing. Red letters indicate to sequencing errors in 'The Arabidopsis Biological Resource Center' (ABRC) server that were confirmed by sequencing of the nMAT3 gene-locus, as well as by BLAST searches against the Brassicales genome resources (see Supplemental Fig. S2).

\section{Supplemental Figure S2. Alignment of nMAT3 intron 1 sequence obtained by blast search of Brassicales genome sequences.}

Alignment was created using the NCBI nucleotide BLASTN server. Arabidopsis thaliana nMAT3 gene (GenBank Accession At5g04050) confirmed the integrity of 3 independent sequencing data (The Hebrew University Genetic Resource). The aligned sequences were downloaded using the BLASTN server.

\section{Supplemental Figure S3. Analysis of the expression of the two-alternative spliced nMAT3 isoforms in Arabidopsis thaliana (Col-0) plants.}

The At5g04050 gene-locus (Fig. S1) harbors two intron sequences that are suggested to be alternatively spliced into two isoforms: annotated as nMAT3.1, encoding a 757 amino acids protein, and the spliced variant, nMAT3.2, encoding a 694 amino acid protein product. The expression of the two putative gene products of At5g04050 was analyzed by RT-PCR with primers flanking introns 1 and 2 (Fig. S3). The unspliced product should yield a 751 nucleotides cDNA product, while the spliced isoform should yield a 500 nucleotide-long mRNA product. The RT-PCR data indicated the existence of only a single isoform, the unspliced nMAT3.1 gene-product in Arabidopsis plants. 


\section{Supplemental Figure S4. $n M A T 3$ gene expression patterns in different tissues and during various developmental stages.}

The expression patterns of nMAT3 were analyzed by publicly available microarray and high throughput sequencing databases, including (a) 'The Arabidopsis Information Resource' (TAIR; http://www.arabidopsis.org) and (b) Genevestigator analysis toolbox (Hruz, et al. 2008, Zimmermann, et al. 2004).

\section{Supplemental Figure S5. Accumulation of mitochondrial transcripts in different nmat mutants.}

Total RNA was extracted from 3-week-old seedlings of wild-type (Col-0) plants and nmat1 (Keren, et al. 2012, Nakagawa and Sakurai 2006), nmat2 (Keren, et al. 2009, Zmudjak, et al. 2017) and nmat 3 mutants. The relative transcript accumulation in the nmat mutants was analyzed by RNA gel blot hybridizations (i.e. northern blot analyses).

\section{Supplemental Figure S6. Accumulation of $n M A T 3$ transcripts in different nmat3 mutants.}

RNA extracted from wild-type (Col-0), embryo-rescued nmat 3 mutants or Col-0 plants at the heart stage (Fig. 3) and nmat3:35S-nMAT3 complemented plants was reverse-transcribed, and the relative steady-state levels of cDNAs corresponding to $n M A T 3$ were evaluated by qPCR with primers which specifically amplified the gene (Supplemental Table S4). The values are means of 9 RT-qPCR reactions corresponding to three biological replicates (error bars indicate one standard deviation), after normalization to the ACTIN2 (At3g1878) and 18S-rRNA (At3g41768) genes.

Supplemental Table S1. Lists of oligonucleotides used for the analysis of the mRNA profiles of wild-type and nmat 3 mutant plants by RT-qPCR experiments.

Supplemental Table S2. Protein profiles quantification data.

Supplemental Table S3. List of antibodies used for the analysis of nmat 3 mutants.

Supplemental Table S4. Oligonucleotides used in screening of individual T-DNA insertion lines in Arabidopsis, and for the cloning of the nMAT3 gene-fusion constructs. 
bioRxiv preprint doi: https://doi.org/10.1101/2020.10.20.346734; this version posted October 21, 2020. The copyright holder for this preprint (which was not certified by peer review) is the author/funder, who has granted bioRxiv a license to display the preprint in perpetuity. It is made available under aCC-BY-ND 4.0 International license.

\section{Author contributions statement}

Sofia Shevtsov-Tal: Plant growth and analysis, embryo rescue and establishment of mutant lines, Biochemical analysis of gene expression, BN-PAGE analysis, RNA isolation, DNA sequencing, analysis of the transcriptome and splicing profiles of Arabidopsis mitochondria by RT-qPCR, respiration analyses. Corinne Best: assisted with RT-qPCR, immunoblots and the analyses of RNA and protein profiles. Roei Matan: assisting in DNA screening and RNA and protein analyses. Dr. Sam Aldrin Chandran: Plant growth and analyses, establishment of mutant lines. Prof. Gregory G. Brown: co-PI and manuscript preparation. Prof. Oren Ostersetzer-Biran: PI, manuscript preparation and corresponding author.

\section{References}

Ahlert, D., Piepenburg, K., Kudla, J. and Bock, R. (2006) Evolutionary origin of a plant mitochondrial group II intron from a reverse transcriptase/maturase-encoding ancestor. $J$ Plant Res, 119, 363-371.

Antonicka, H., Ogilvie, I., Taivassalo, T., Anitori, R.P., Haller, R.G., Vissing, J., Kennaway, N.G. and Shoubridge, E.A. (2003) Identification and characterization of a common set of complex I assembly intermediates in mitochondria from patients with complex I deficiency. J Biol Chem, 278, 43081-43088.

Aryamanesh, N., Ruwe, H., Sanglard, L.V.P., Eshraghi, L., Bussell, J.D., Howell, K.A., Small, I. and des Francs-Small, C.C. (2017) The pentatricopeptide repeat protein EMB2654 is essential for trans-splicing of a chloroplast small ribosomal subunit transcript. Plant Physiol, 173, 1164-1176.

Baradaran, R., Berrisford, J.M., Minhas, G.S. and Sazanov, L.A. (2013) Crystal structure of the entire respiratory complex I. Nature, 494, 443-448.

Barkan, A. and Small, I. (2014) Pentatricopeptide Repeat Proteins in Plants. Annu Rev Plant Biol, 65, 415-442.

Best, C., Mizrahi, R. and Ostersetzer-Biran, O. (2020) Why so Complex? The Intricacy of Genome Structure and Gene Expression, Associated with Angiosperm Mitochondria, May Relate to the Regulation of Embryo Quiescence or Dormancy-Intrinsic Blocks to Early Plant Life. Plants (Basel, Switzerland), 9, 1-19.

Best, C., Zmudjak, M. and Ostersetzer, O. (2019) The PPR-related splicing cofactor MSP1/EMB1025 protein, encoded by At4g20090, encode an essential protein that is required for the splicing of nad1 intron 1 and for the biogenesis of complex $I$ in Arabidopsis mitochondria. bioRxiv, 615856 .

Bonen, L. (2018) Mitochondrial Genomes in Land Plants. In Molecular Life Sciences: An Encyclopedic Reference (Wells, R.D., Bond, J.S., Klinman, J. and Masters, B.S.S. eds). New York, NY: Springer New York, pp. 734-742.

Boyes, D.C., Zayed, A.M., Ascenzi, R., McCaskill, A.J., Hoffman, N.E., Davis, K.R. and Görlach, J. (2001) Growth Stage-Based Phenotypic Analysis of Arabidopsis. A Model for High Throughput Functional Genomics in Plants. The Plant cell, 13, 1499-1510.

Braun, H.-P., Binder, S., Brennicke, A., Eubel, H., Fernie, A.R., Finkemeier, I., Klodmann, J., König, A.-C., Kühn, K., Meyer, E., Obata, T., Schwarzländer, M., Takenaka, M. and Zehrmann, A. (2014) The life of plant mitochondrial complex I. Mitochondrion, 19, Part B, 295-313. 
bioRxiv preprint doi: https://doi.org/10.1101/2020.10.20.346734; this version posted October $21,2020$. The copyright holder for this preprint (which was not certified by peer review) is the author/funder, who has granted bioRxiv a license to display the preprint in perpetuity. It is made available under aCC-BY-ND 4.0 International license.

Brown, G.G., Colas des Francs-Small, C. and Ostersetzer-Biran, O. (2014) Group II intron splicing factors in plant mitochondria. Frontiers in plant science, 5, 35.

Cardol, P., Matagne, R.F. and Remacle, C. (2002) Impact of mutations affecting ND mitochondria-encoded subunits on the activity and assembly of complex I in Chlamydomonas. Implication for the structural organization of the enzyme. J Mol Biol, 319, 1211-1221.

Clough, S.J. and Bent, A.F. (1998) Floral dip: a simplified method for Agrobacteriummediated transformation of Arabidopsis thaliana. Plant J, 16, 735-743.

Cohen, S., Zmudjak, M., Colas des Francs-Small, C., Malik, S., Shaya, F., Keren, I., Belausov, E., Many, Y., Brown, G.G., Small, I. and Ostersetzer-Biran, O. (2014) nMAT4, a maturase factor required for nad 1 pre-mRNA processing and maturation, is essential for holocomplex I biogenesis in Arabidopsis mitochondria. The Plant Journal, 78, 253-268.

Colas des Francs-Small, C., Kroeger, T., Zmudjak, M., Ostersetzer-Biran, O., Rahimi, N., Small, I. and Barkan, A. (2012) A PORR domain protein required for rpl2 and ccmFc intron splicing and for the biogenesis of c-type cytochromes in Arabidopsis mitochondria. Plant $J$, 69, 996-1005.

Colas des Francs-Small, C. and Small, I. (2014) Surrogate mutants for studying mitochondrially encoded functions. Biochimie, 100, 234-242.

Cordoba, J.P., Marchetti, F., Soto, D., Martin, M.V., Pagnussat, G.C. and Zabaleta, E. (2016) The CA domain of the respiratory complex I is required for normal embryogenesis in Arabidopsis thaliana. J Exp Bot, 67, 1589-1603.

Dahan, J., Tcherkez, G., Macherel, D., Benamar, A., Belcram, K., Quadrado, M., Arnal, N. and Mireau, H. (2014) Disruption of the CYTOCHROME C OXIDASE DEFICIENT1 gene leads to cytochrome c oxidase depletion and reorchestrated respiratory metabolism in Arabidopsis. Plant Physiol, 166, 1788-1802.

Despres, B., Delseny, M. and Devic, M. (2001) Partial complementation of embryo defective mutations: a general strategy to elucidate gene function. Plant J, 27, 149-159.

Dlakic, M. and Mushegian, A. (2011) Prp8, the pivotal protein of the spliceosomal catalytic center, evolved from a retroelement-encoded reverse transcriptase. $R N A, \mathbf{1 7}, 799-808$.

Dutilleul, C., Driscoll, S., Cornic, G., De Paepe, R., Foyer, C.H. and Noctor, G. (2003) Functional mitochondrial complex I is required by tobacco leaves for optimal photosynthetic performance in photorespiratory conditions and during transients. Plant Physiol, 131, 264275.

Falcon de Longevialle, A., Meyer, E.H., Andres, C., Taylor, N.L., Lurin, C., Millar, A.H. and Small, I.D. (2007) The pentatricopeptide repeat gene OTP43 is required for transsplicing of the mitochondrial nadl intron 1 in Arabidopsis thaliana. Plant Cell, 19, 32563265.

Focks, N. and Benning, C. (1998) wrinkled1: A novel, low-seed-oil mutant of Arabidopsis with a deficiency in the seed-specific regulation of carbohydrate metabolism. Plant Physiol, 118, 91-101.

Fourquin, C., Beauzamy, L., Chamot, S., Creff, A., Goodrich, J., Boudaoud, A. and Ingram, G. (2016) Mechanical stress mediated by both endosperm softening and embryo growth underlies endosperm elimination in $<\mathrm{em}>$ Arabidopsis $</$ em $>$ seeds. Development, 143, 3300-3305.

Franzmann, L., Patton, D.A. and Meinke, D.W. (1989) In vitro morphogenesis of arrested embryos from lethal mutants of Arabidopsis thaliana. Theoretical and Applied Genetics, 77, 609-616.

Fromm, S., Going, J., Lorenz, C., Peterhansel, C. and Braun, H.P. (2016a) Depletion of the "gamma-type carbonic anhydrase-like" subunits of complex I affects central mitochondrial metabolism in Arabidopsis thaliana. Biochim Biophys Acta, 1857, 60-71. 
bioRxiv preprint doi: https://doi.org/10.1101/2020.10.20.346734; this version posted October $21,2020$. The copyright holder for this preprint (which was not certified by peer review) is the author/funder, who has granted bioRxiv a license to display the preprint in perpetuity. It is made available under aCC-BY-ND 4.0 International license.

Fromm, S., Senkler, J., Eubel, H., Peterhansel, C. and Braun, H.P. (2016b) Life without complex I: proteome analyses of an Arabidopsis mutant lacking the mitochondrial NADH dehydrogenase complex. J Exp Bot, 67, 3079-3093.

Fuchs, P., Rugen, N., Carrie, C., Elsasser, M., Finkemeier, I., Giese, J., Hildebrandt, T.M., Kuhn, K., Maurino, V.G., Ruberti, C., Schallenberg-Rudinger, M., Steinbeck, J., Braun, H.P., Eubel, H., Meyer, E.H., Muller-Schussele, S.J. and Schwarzlander, M. (2020) Single organelle function and organization as estimated from Arabidopsis mitochondrial proteomics. Plant J, 101, 420-441.

Grewe, F., Edger, P.P., Keren, I., Sultan, L., Pires, J.C., Ostersetzer-Biran, O. and Mower, J.P. (2014) Comparative analysis of 11 Brassicales mitochondrial genomes and the mitochondrial transcriptome of Brassica oleracea. Mitochondrion, 19, 135-143.

Gualberto, J.M., Le Ret, M., Beator, B. and Kuhn, K. (2015) The RAD52-like protein ODB1 is required for the efficient excision of two mitochondrial introns spliced via first-step hydrolysis. Nucleic Acids Res, 43, 6500-6510.

Gualberto, J.M. and Newton, K.J. (2017) Plant mitochondrial genomes: Dynamics and mechanisms of mutation. Annu Rev Plant Biol, 68, 225-252.

Guo, W., Grewe, F., Fan, W., Young, G.J., Knoop, V., Palmer, J.D. and Mower, J.P. (2016) Ginkgo and Welwitschia Mitogenomes Reveal Extreme Contrasts in Gymnosperm Mitochondrial Evolution. Mol Biol Evol, 33, 1448-1460.

Hammani, K. and Giege, P. (2014) RNA metabolism in plant mitochondria. Trends Plant Sci, 19, 380-389.

He, J., Duan, Y., Hua, D., Fan, G., Wang, L., Liu, Y., Chen, Z., Han, L., Qu, L.-J. and Gong, Z. (2012) DEXH box RNA helicase-mediated mitochondrial reactive oxygen species production in Arabidopsis mediates crosstalk between abscisic acid and auxin signaling. The Plant Cell, 24, 1815-1833.

Hooper, C.M., Castleden, I.R., Tanz, S.K., Aryamanesh, N. and Millar, A.H. (2017) SUBA4: the interactive data analysis centre for Arabidopsis subcellular protein locations. Nucleic acids research, 45, D1064-D1074.

Hruz, T., Laule, O., Szabo, G., Wessendorp, F., Bleuler, S., Oertle, L., Widmayer, P., Gruissem, W. and Zimmermann, P. (2008) Genevestigator V3: a reference expression database for the meta-analysis of transcriptomes. Adv Bioinformatics, 2008, 420747-420751.

Jacoby, R.P., Li, L., Huang, S., Pong Lee, C., Millar, A.H. and Taylor, N.L. (2012) Mitochondrial composition, function and stress response in plants. J Integr Plant Biol, 54, 887-906.

Johnson, K.L., Faulkner, C., Jeffree, C.E. and Ingram, G.C. (2008) The Phytocalpain Defective Kernel 1 Is a Novel $<$ em $>$ Arabidopsis $</$ em $>$ Growth Regulator Whose Activity Is Regulated by Proteolytic Processing. The Plant Cell, 20, 2619-2630.

Keren, I., Bezawork-Geleta, A., Kolton, M., Maayan, I., Belausov, E., Levy, M., Mett, A., Gidoni, D., Shaya, F. and Ostersetzer-Biran, O. (2009) AtnMat2, a nuclear-encoded maturase required for splicing of group-II introns in Arabidopsis mitochondria. $R N A, \mathbf{1 5}$, 2299-2311.

Keren, I., Shaya, F. and Ostersetzer-Biran, O. (2011) An optimized method for the analysis of plant mitochondria RNAs by northern-blotting. Endocy Cell Res, 1, 34-42.

Keren, I., Tal, L., Colas des Francs-Small, C., Araújo, W.L., Shevtsov, S., Shaya, F., Fernie, A.R., Small, I. and Ostersetzer-Biran, O. (2012) nMAT1, a nuclear-encoded maturase involved in the trans-splicing of nadl intron 1, is essential for mitochondrial complex I assembly and function. The Plant Journal, 71, 413-426.

Kleine, T. and Leister, D. (2016) Retrograde signaling: Organelles go networking. Biochim Biophys Acta, 1857, 1313-1325. 
bioRxiv preprint doi: https://doi.org/10.1101/2020.10.20.346734; this version posted October $21,2020$. The copyright holder for this preprint (which was not certified by peer review) is the author/funder, who has granted bioRxiv a license to display the preprint in perpetuity. It is made available under aCC-BY-ND 4.0 International license.

Klodmann, J., Sunderhaus, S., Nimtz, M., Jänsch, L. and Braun, H.-P. (2010) Internal architecture of mitochondrial complex I from Arabidopsis thaliana. Plant Cell, 22, 797-810.

Köhler, D., Schmidt-Gattung, S. and Binder, S. (2010) The DEAD-box protein PMH2 is required for efficient group II intron splicing in mitochondria of Arabidopsis thaliana. Plant Mol Biol, 72, 459-467.

Kuhn, K., Obata, T., Feher, K., Bock, R., Fernie, A.R. and Meyer, E.H. (2015) Complete Mitochondrial Complex I Deficiency Induces an Up-Regulation of Respiratory Fluxes That Is Abolished by Traces of Functional Complex I. Plant Physiol, 168, 1537-1549.

Laemmli, U.K. (1970) Cleavage of structural proteins during the assembly of the head of bacteriophage T4. Nature, 227, 680-685.

Lamattina, L., Gonzalez, D., Gualberto, J. and Grienenberger, J.-M. (1993) Higher plant mitochondria encode an homologue of the nuclear-encoded $30-\mathrm{kDa}$ subunit of bovine mitochondrial complex I. Eur J Biochem, 217, 831-838.

Lambowitz, A.M. and Zimmerly, S. (2011) Group II introns: mobile ribozymes that invade DNA. Perspect Biol, 3, 1-19.

Lee, C.P., Taylor, N.L. and Millar, A.H. (2013) Recent advances in the composition and heterogeneity of the Arabidopsis mitochondrial proteome. Frontiers in plant science, 4.

Lee, K., Han, J.H., Park, Y.I., Colas des Francs-Small, C., Small, I. and Kang, H. (2017) The mitochondrial pentatricopeptide repeat protein PPR19 is involved in the stabilization of NADH dehydrogenase 1 transcripts and is crucial for mitochondrial function and Arabidopsis thaliana development. The New phytologist.

Letunic, I., Doerks, T. and Bork, P. (2012) SMART 7: recent updates to the protein domain annotation resource. Nucleic Acids Res, 40, D302-305.

Li-Pook-Than, J., Carrillo, C. and Bonen, L. (2004) Variation in mitochondrial transcript profiles of protein-coding genes during early germination and seedling development in wheat. Current Genetics, V46, 374.

Ligas, J., Pineau, E., Bock, R., Huynen, M.A. and Meyer, E.H. (2019) The assembly pathway of complex I in Arabidopsis thaliana. The Plant Journal, 97, 447-459.

Maclean, A.E., Hertle, A.P., Ligas, J., Bock, R., Balk, J. and Meyer, E.H. (2018) Absence of complex I is associated with diminished respiratory chain function in European mistletoe. Curr Biol, 28, 1614-1619.e1613.

Marchler-Bauer, A., Anderson, J.B., DeWeese-Scott, C., Fedorova, N.D., Geer, L.Y., He, S., Hurwitz, D.I., Jackson, J.D., Jacobs, A.R., Lanczycki, C.J., Liebert, C.A., Liu, C., Madej, T., Marchler, G.H., Mazumder, R., Nikolskaya, A.N., Panchenko, A.R., Rao, B.S., Shoemaker, B.A., Simonyan, V., Song, J.S., Thiessen, P.A., Vasudevan, S., Wang, Y., Yamashita, R.A., Yin, J.J. and Bryant, S.H. (2003) CDD: a curated Entrez database of conserved domain alignments. Nucl. Acids Res., 31, 383-387.

Matsuura, M., Noah, J.W. and Lambowitz, A.M. (2001) Mechanism of maturase-promoted group II intron splicing. EMBO J, 20, 7259-7270.

Meyer, E.H. (2012) Proteomic investigations of complex I composition: how to define a subunit? Frontiers in plant science, 3, 106.

Meyer, E.H., Solheim, C., Tanz, S.K., Bonnard, G. and Millar, A.H. (2011) Insights into the composition and assembly of the membrane arm of plant complex I through analysis of subcomplexes in Arabidopsis mutant lines. J Biol Chem, 286, 26081-26092.

Meyer, E.H., Tomaz, T., Carroll, A.J., Estavillo, G., Delannoy, E., Tanz, S.K., Small, I.D., Pogson, B.J. and Millar, A.H. (2009) Remodeled respiration in ndufs4 with low phosphorylation efficiency suppresses Arabidopsis germination and growth and alters control of metabolism at night. Plant Physiol, 151, 603-619.

Millar, A.H., Whelan, J., Soole, K.L. and Day, D.A. (2011) Organization and regulation of mitochondrial respiration in plants. Ann Rev Plant Biol, 62, 79-104. 
bioRxiv preprint doi: https://doi.org/10.1101/2020.10.20.346734; this version posted October $21,2020$. The copyright holder for this preprint (which was not certified by peer review) is the author/funder, who has granted bioRxiv a license to display the preprint in perpetuity. It is made available under aCC-BY-ND 4.0 International license.

Mizzotti, C., Rotasperti, L., Moretto, M., Tadini, L., Resentini, F., Galliani, B.M., Galbiati, M., Engelen, K., Pesaresi, P. and Masiero, S. (2018) Time-Course Transcriptome Analysis of Arabidopsis Siliques Discloses Genes Essential for Fruit Development and Maturation. Plant Physiol, 178, 1249-1268.

Mohr, G. and Lambowitz, A.M. (2003) Putative proteins related to group II intron reverse transcriptase/maturases are encoded by nuclear genes in higher plants. Nucl Acids Res, 31, 647-652.

Mohr, G., Perlman, P.S. and Lambowitz, A.M. (1993) Evolutionary relationships among group II intron-encoded proteins and identification of a conserved domain that may be related to maturase function. Nucleic Acids Research, 21, 4991-4997.

Mohr, S., Matsuura, M., Perlman, P.S. and Lambowitz, A.M. (2006) A DEAD-box protein alone promotes group II intron splicing and reverse splicing by acting as an RNA chaperone. PNAS, 103, 3569-3574.

Mower, J.P., Sloan, D.B. and Alverson, A.J. (2012) Plant mitochondrial genome diversity: the genomics revolution. In: Plant Genome Diversity Volume 1: Plant Genomes, their Residents, and their Evolutionary Dynamics. Wendel JF, Greilhuber J, Dolezel J, Leitch IJ (eds). Springer:Dordrecht. pp 123-144.

Nakagawa, N. and Sakurai, N. (2006) A mutation in At-nMatla, which encodes a nuclear gene having high similarity to group II Intron maturase, causes impaired splicing of mitochondrial nad4 transcript and altered carbon metabolism in Arabidopsis thaliana. Plant Cell Physiol, 47, 772-783.

Ostersetzer-Biran, O. (2016) Respiratory complex I and embryo development. J Exp Bot, 67, 1205-1207.

Ostersetzer, O., Cooke, A.M., Watkins, K.P. and Barkan, A. (2005) CRS1, a chloroplast group II intron splicing factor, promotes intron folding through specific interactions with two intron domains. Plant Cell, 17, 241-255.

Park, S., Grewe, F., Zhu, A., Ruhlman, T.A., Sabir, J., Mower, J.P. and Jansen, R.K. (2015) Dynamic evolution of Geranium mitochondrial genomes through multiple horizontal and intracellular gene transfers. The New phytologist, 208, 570-583.

Perales, M., Eubel, H., Heinemeyer, J., Colaneri, A., Zabaleta, E. and Braun, H.-P. (2005) Disruption of a nuclear gene encoding a mitochondrial gamma carbonic anhydrase reduces complex I and supercomplex I+III2 levels and alters mitochondrial physiology in Arabidopsis. J Mol Biol, 350, 263-277.

Petersen, G., Cuenca, A., Moller, I.M. and Seberg, O. (2015) Massive gene loss in mistletoe (Viscum, Viscaceae) mitochondria. Sci Rep, 5, 1-7.

Pineau, B., Layoune, O., Danon, A. and De Paepe, R. (2008) L-galactono-1,4-lactone dehydrogenase is required for the accumulation of plant respiratory complex I. $J$ Biol Chem, 283, 32500-32505.

Pinfield-Wells, H., Rylott, E.L., Gilday, A.D., Graham, S., Job, K., Larson, T.R. and Graham, I.A. (2005) Sucrose rescues seedling establishment but not germination of Arabidopsis mutants disrupted in peroxisomal fatty acid catabolism. Plant J, 43, 861-872.

Priault, P., Fresneau, C., Noctor, G., De Paepe, R., Cornic, G. and Streb, P. (2006) The mitochondrial CMSII mutation of Nicotiana sylvestris impairs adjustment of photosynthetic carbon assimilation to higher growth irradiance. Journal of Experimental Botany, 57, 20752085.

Radchuk, V. and Borisjuk, L. (2014) Physical, metabolic and developmental functions of the seed coat. Frontiers in plant science, 5, 510-510.

Schertl, P. and Braun, H.P. (2014) Respiratory electron transfer pathways in plant mitochondria. Frontiers in plant science, 5, 163. 
bioRxiv preprint doi: https://doi.org/10.1101/2020.10.20.346734; this version posted October $21,2020$. The copyright holder for this preprint (which was not certified by peer review) is the author/funder, who has granted bioRxiv a license to display the preprint in perpetuity. It is made available under aCC-BY-ND 4.0 International license.

Schmitz-Linneweber, C., Lampe, M.-K., Sultan, L.D. and Ostersetzer-Biran, O. (2015) Organellar maturases: A window into the evolution of the spliceosome. BBA - Bioenergetics, 1847, 798-808.

Senkler, J., Rugen, N., Eubel, H., Hegermann, J. and Braun, H.-P. (2018) Absence of complex I implicates rearrangement of the respiratory chain in European mistletoe. Curr Biol, 28, 1606-1613.e1604.

Senkler, J., Senkler, M., Eubel, H., Hildebrandt, T., Lengwenus, C., Schertl, P., Schwarzländer, M., Wagner, S., Wittig, I. and Braun, H.-P. (2017) The mitochondrial complexome of Arabidopsis thaliana. Plant J, 89, 1079-1092.

Shevtsov, S., Nevo-Dinur, K., Faigon, L., Sultan, L.D., Zmudjak, M., Markovits, M. and Ostersetzer-Biran, O. (2018) Control of organelle gene expression by the mitochondrial transcription termination factor mTERF22 in Arabidopsis thaliana plants. PLOS ONE, 13, e0201631.

Sloan, D., Alverson, A., Chuckalovcak, J., Wu, M., McCauley, D., Palmer, J. and Taylor, D. (2012) Rapid evolution of enormous, multichromosomal genomes in flowering plant mitochondria with exceptionally high mutation rates. PLoS Biol, 10, e1001241.

Sloan, D.B., Wu, Z. and Sharbrough, J. (2018) Correction of Persistent Errors in Arabidopsis Reference Mitochondrial Genomes. The Plant Cell, 30, 525-527.

Small, I.D., Schallenberg-Rudinger, M., Takenaka, M., Mireau, H. and OstersetzerBiran, O. (2020) Plant organellar RNA editing: what 30 years of research has revealed. Plant $J$, 101, 1040-1056.

Soufari, H., Parrot, C., Kuhn, L., Waltz, F. and Hashem, Y. (2020) Specific features and assembly of the plant mitochondrial complex I revealed by cryo-EM. bioRxiv, 2020.2002.2021.959148.

Subrahmanian, N., Remacle, C. and Hamel, P.P. (2016) Plant mitochondrial complex I composition and assembly: A review. Biochimica et Biophysica Acta (BBA) - Bioenergetics, 1857, 1001-1014.

Sultan, L.D., Mileshina, D., Grewe, F., Rolle, K., Abudraham, S., Glodowicz, P., Khan Niazi, A., keren, I., Shevtsov, S., Klipcan, L., Barciszewski, J., Mower, J.P., Dietrich, A. and Ostersetzer, O. (2016) The reverse-transcriptase/RNA-maturase protein MatR is required for the splicing of various group II introns in Brassicaceae mitochondria. The Plant Cell, 28, 2805-2829.

Sun, Y.K., Gutmann, B., Yap, A., Kindgren, P. and Small, I. (2018) Editing of Chloroplast rps14 by PPR Editing Factor EMB2261 Is Essential for Arabidopsis Development. Frontiers in plant science, 9, 1-11.

Sunderhaus, S., Dudkina, N.V., Jansch, L., Klodmann, J., Heinemeyer, J., Perales, M., Zabaleta, E., Boekema, E.J. and Braun, H.P. (2006) Carbonic anhydrase subunits form a matrix-exposed domain attached to the membrane arm of mitochondrial complex I in plants. J Biol Chem, 281, 6482-6488.

Sunderhaus, S., Klodmann, J., Lenz, C. and Braun, H.-P. (2010) Supramolecular structure of the OXPHOS system in highly thermogenic tissue of Arum maculatum. Plant Physiol Bioch, 48, 265-272.

Unseld, M., Marienfeld, J.R., Brandt, P. and Brennicke, A. (1997) The mitochondrial genome of Arabidopsis thaliana contains 57 genes in 366,924 nucleotides. Nature Genetics, 15, 57-61.

Wahleithner, J.A., MacFarlane, J.L. and Wolstenholme, D.R. (1990) A sequence encoding a maturase-related protein in a group II intron of a plant mitochondrial nad1 gene. PNAS, 87, $548-552$. 
bioRxiv preprint doi: https://doi.org/10.1101/2020.10.20.346734; this version posted October 21, 2020. The copyright holder for this preprint (which was not certified by peer review) is the author/funder, who has granted bioRxiv a license to display the preprint in perpetuity. It is made available under aCC-BY-ND 4.0 International license.

Wang, C., Aube, F., Planchard, N., Quadrado, M., Dargel-Graffin, C., Nogue, F. and Mireau, H. (2017) The pentatricopeptide repeat protein MTSF2 stabilizes a nad1 precursor transcript and defines the 3 end of its 5-half intron. Nucleic Acids Res.

Wang, C., Aube, F., Quadrado, M., Dargel-Graffin, C. and Mireau, H. (2018) Three new pentatricopeptide repeat proteins facilitate the splicing of mitochondrial transcripts and complex I biogenesis in Arabidopsis. J Exp Bot, 69, 5131-5140.

Woodson, J.D. and Chory, J. (2008) Coordination of gene expression between organellar and nuclear genomes. Nat Rev Genet, 9, 383-395.

Zimmerly, S. and Semper, C. (2015) Evolution of group II introns. Mobile DNA, 6, 1-19.

Zimmermann, P., Hirsch-Hoffmann, M., Hennig, L. and Gruissem, W. (2004) GENEVESTIGATOR. Arabidopsis microarray database and analysis toolbox. Plant Physiol, 136, 2621-2632.

Zmudjak, M., Colas des Francs-Small, C., Keren, I., Shaya, F., Belausov, E., Small, I. and Ostersetzer-Biran, O. (2013) mCSF1, a nucleus-encoded CRM protein required for the processing of many mitochondrial introns, is involved in the biogenesis of respiratory complexes I and IV in Arabidopsis. New Phytologist, 199, 379-394.

Zmudjak, M. and Ostersetzer-Biran, O. (2017) RNA metabolism and transcript regulation. In Annual Plant Reviews, Volume 50 pp. 261-309, D. C. Logan (Ed.).

Zmudjak, M., Shevtsov, S., Sultan, L.D., Keren, I. and Ostersetzer-Biran, O. (2017) Analysis of the roles of the Arabidopsis nMAT2 and PMH2 proteins provided with new insights into the regulation of group II intron splicing in land-plant mitochondria. Int $\mathrm{J} \mathrm{Mol}$ Sci, 18, 1-25. 
a Intron encoded maturases in nature

Canonical type II ORFs

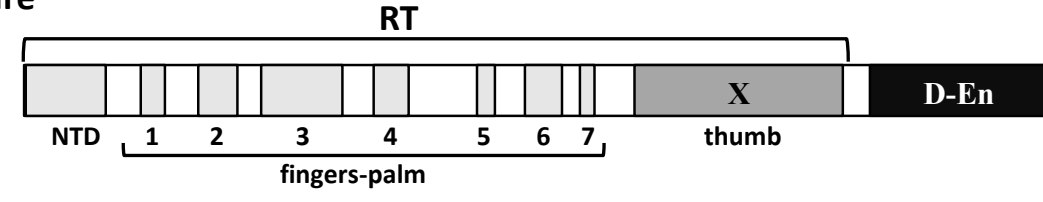

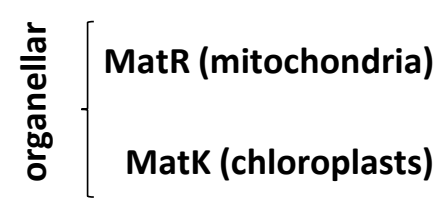
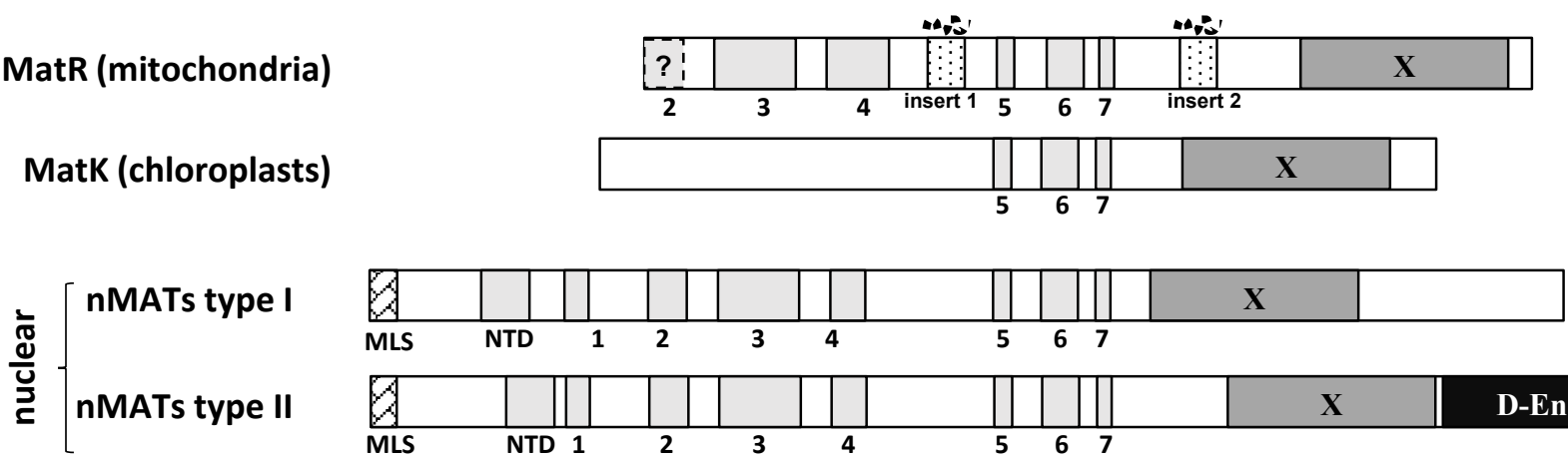

\section{b nMAT3 (At5g04050) gene-locus}
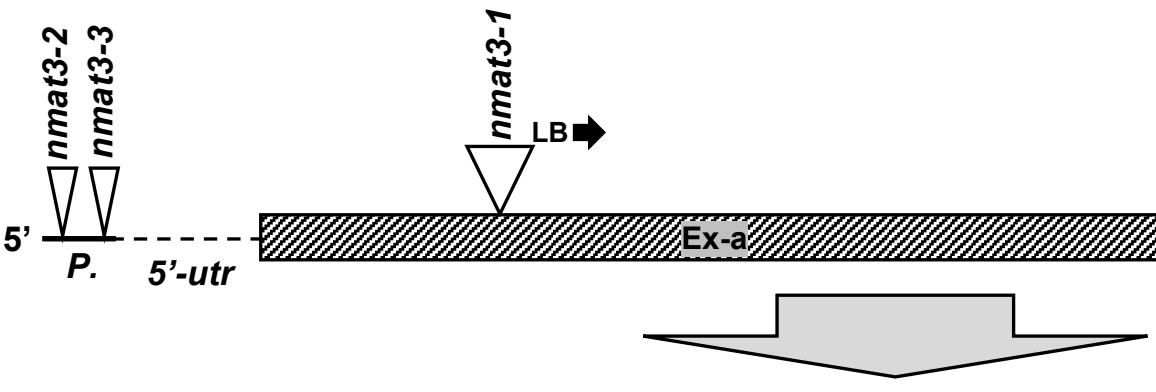

nMAT3 isoforms

RT

nMAT3.1 $\mathrm{NH}_{3}{ }^{\prime}-$

fingers-palm

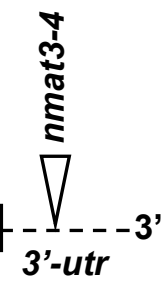

$100 \mathrm{nts}$

nMAT3.2 $\mathrm{NH}_{3}{ }^{\prime}-$

fingers-plam

100 aa

\section{C genotyping nmat3 mutants by PCR}

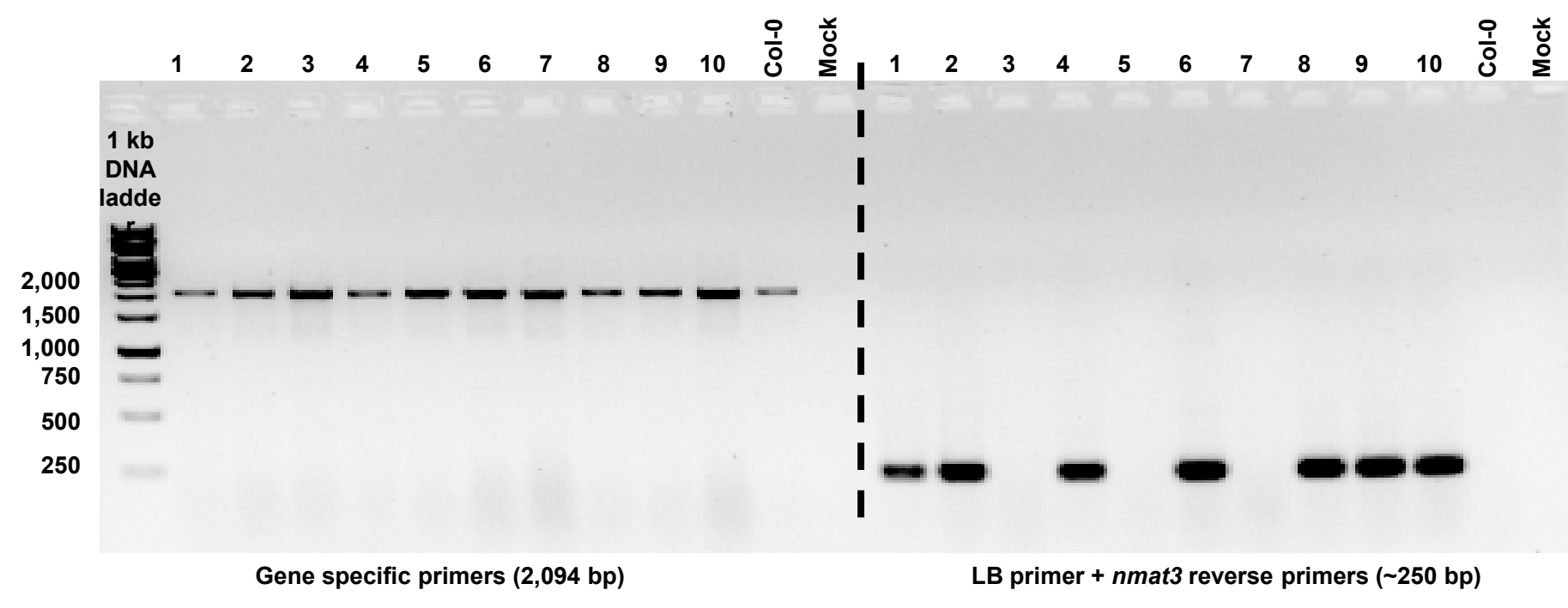

Figure 1 


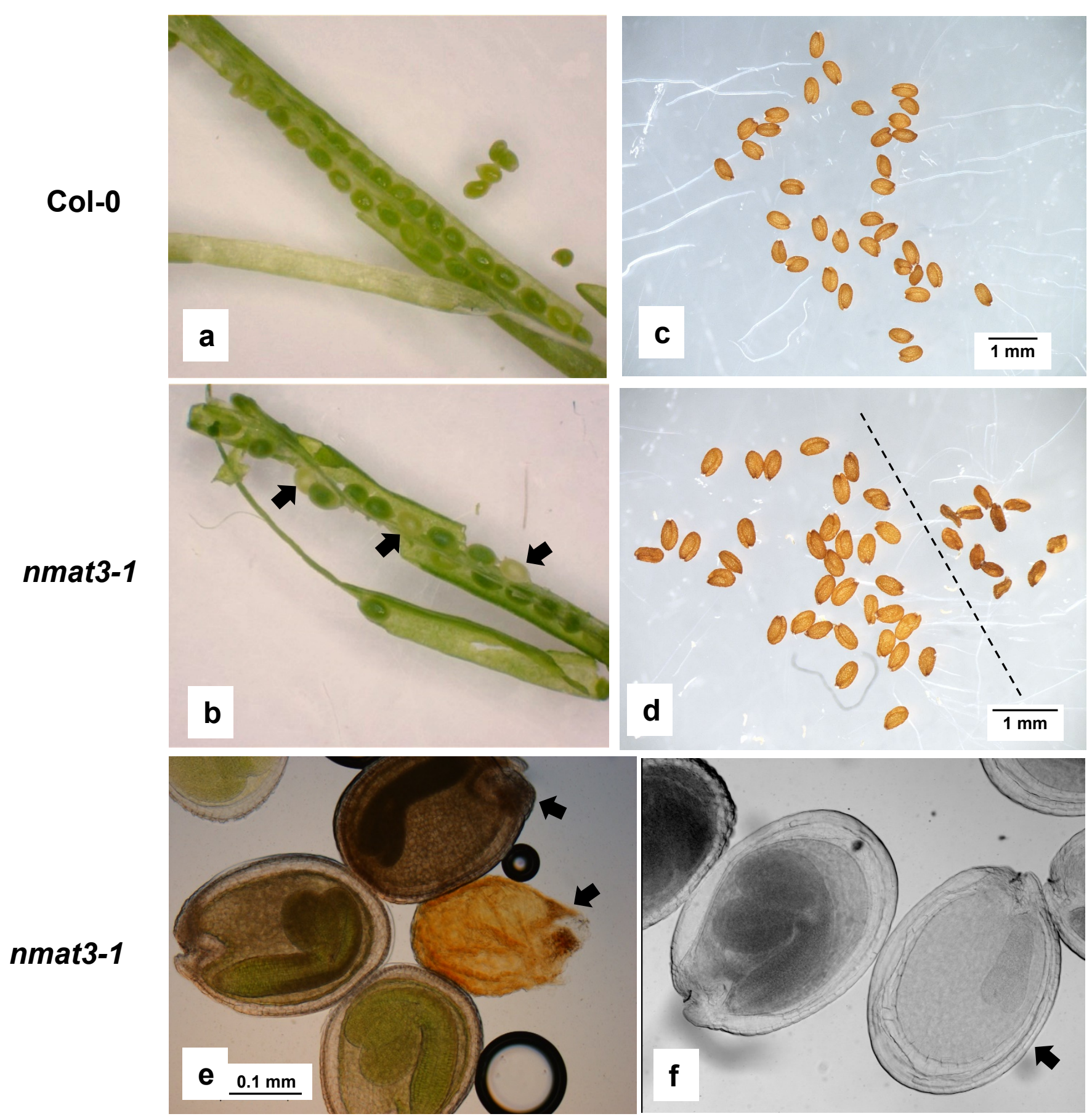

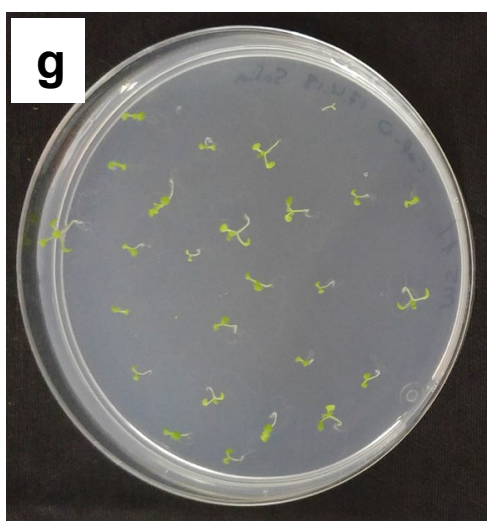

Col-0

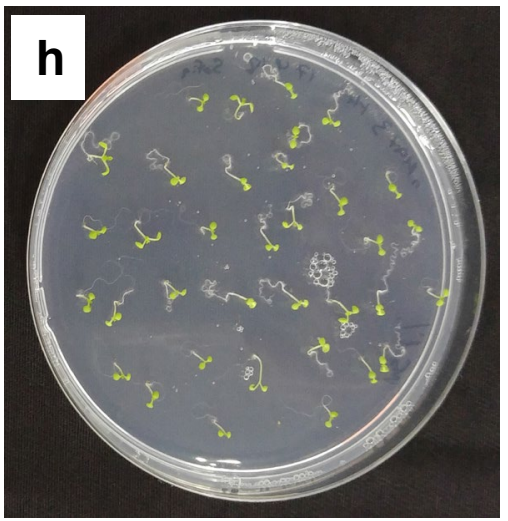

normal

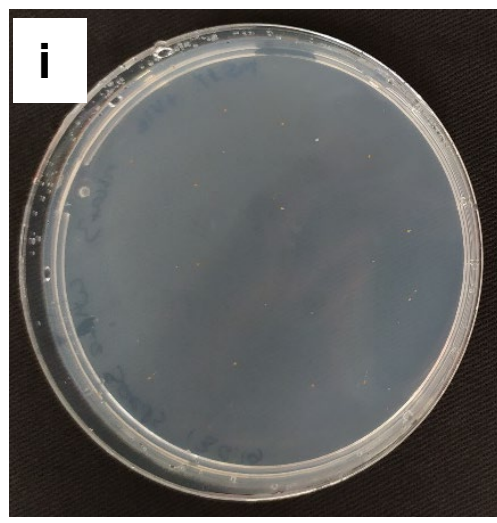

wrinkled

Figure 2 
$\square$ nmat3-1 $\square$ Col-0 heart

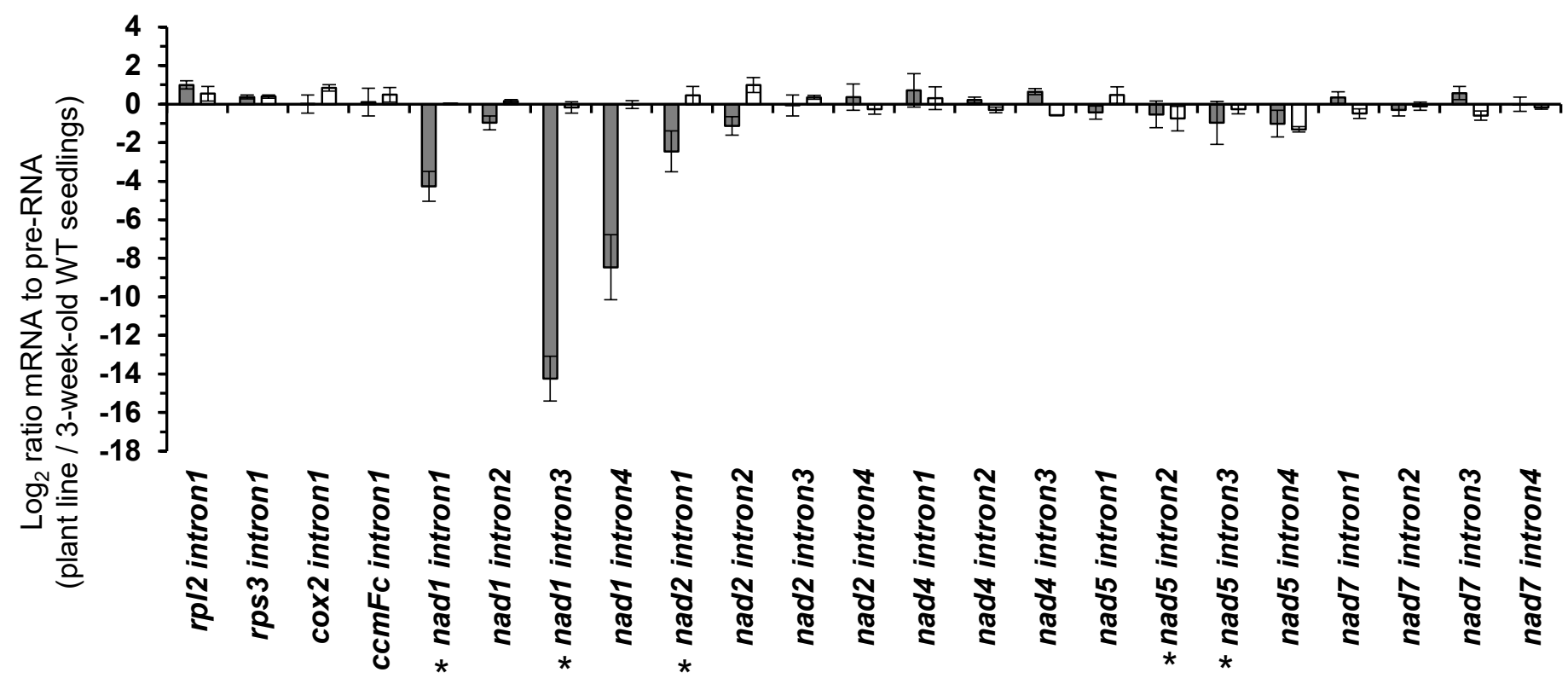

b

\$Heterozygous $\square 35$ S:nMAT3

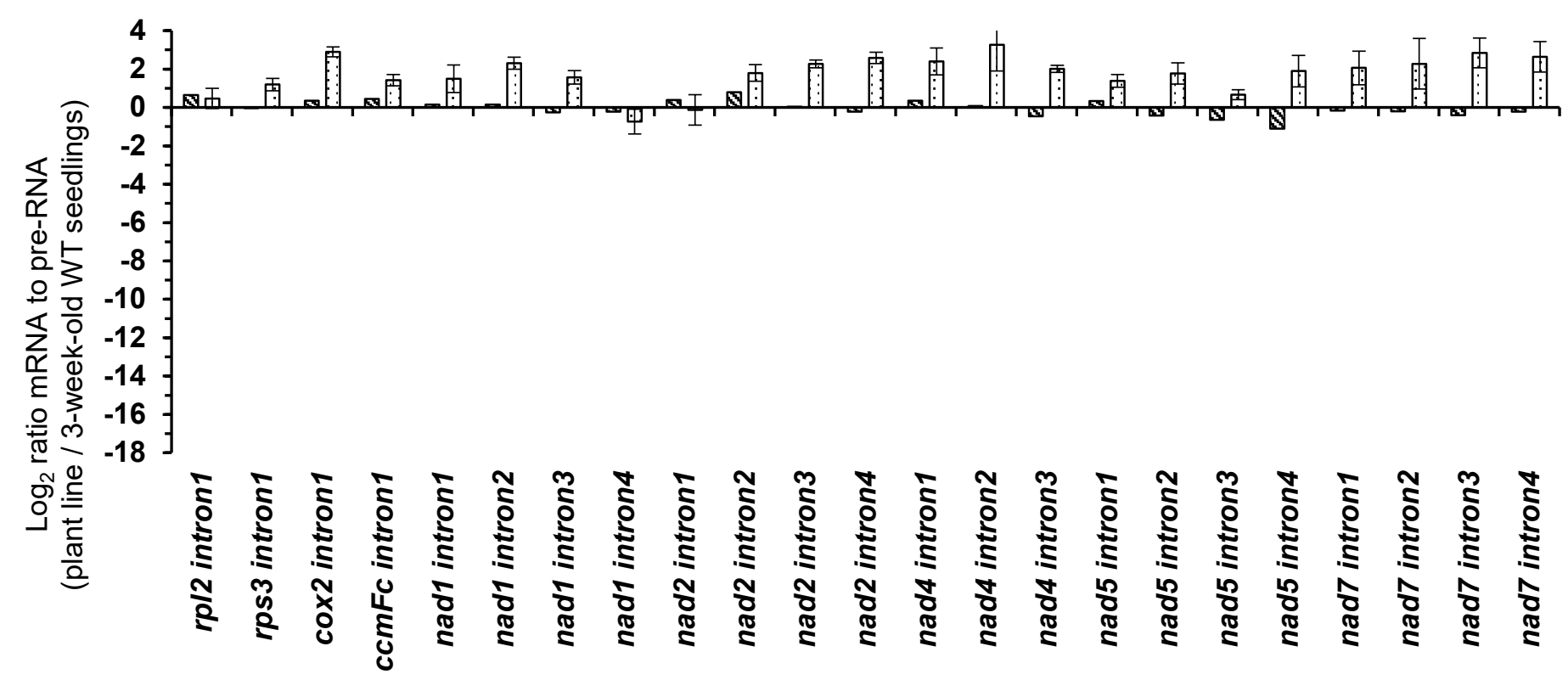

Figure 5 

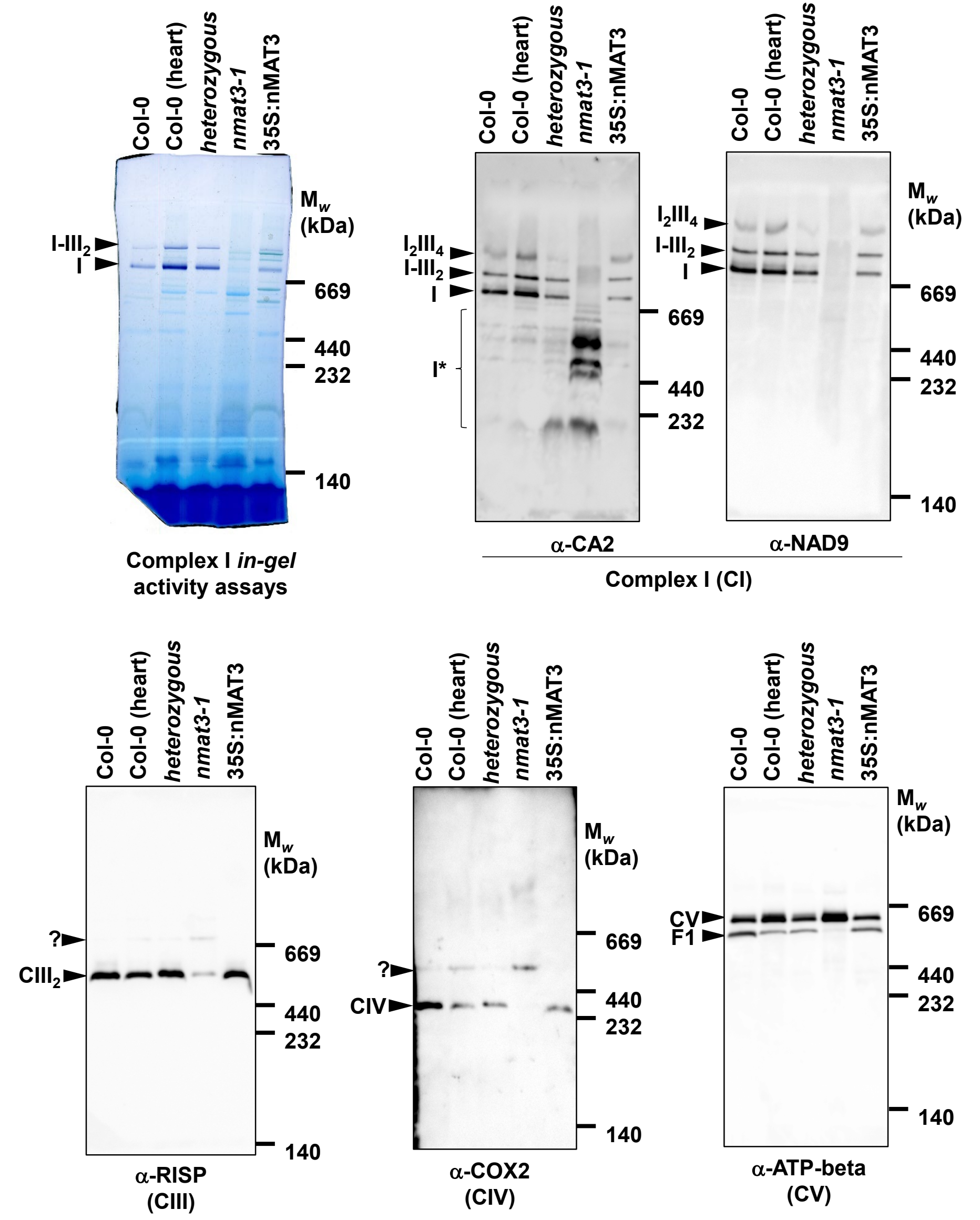


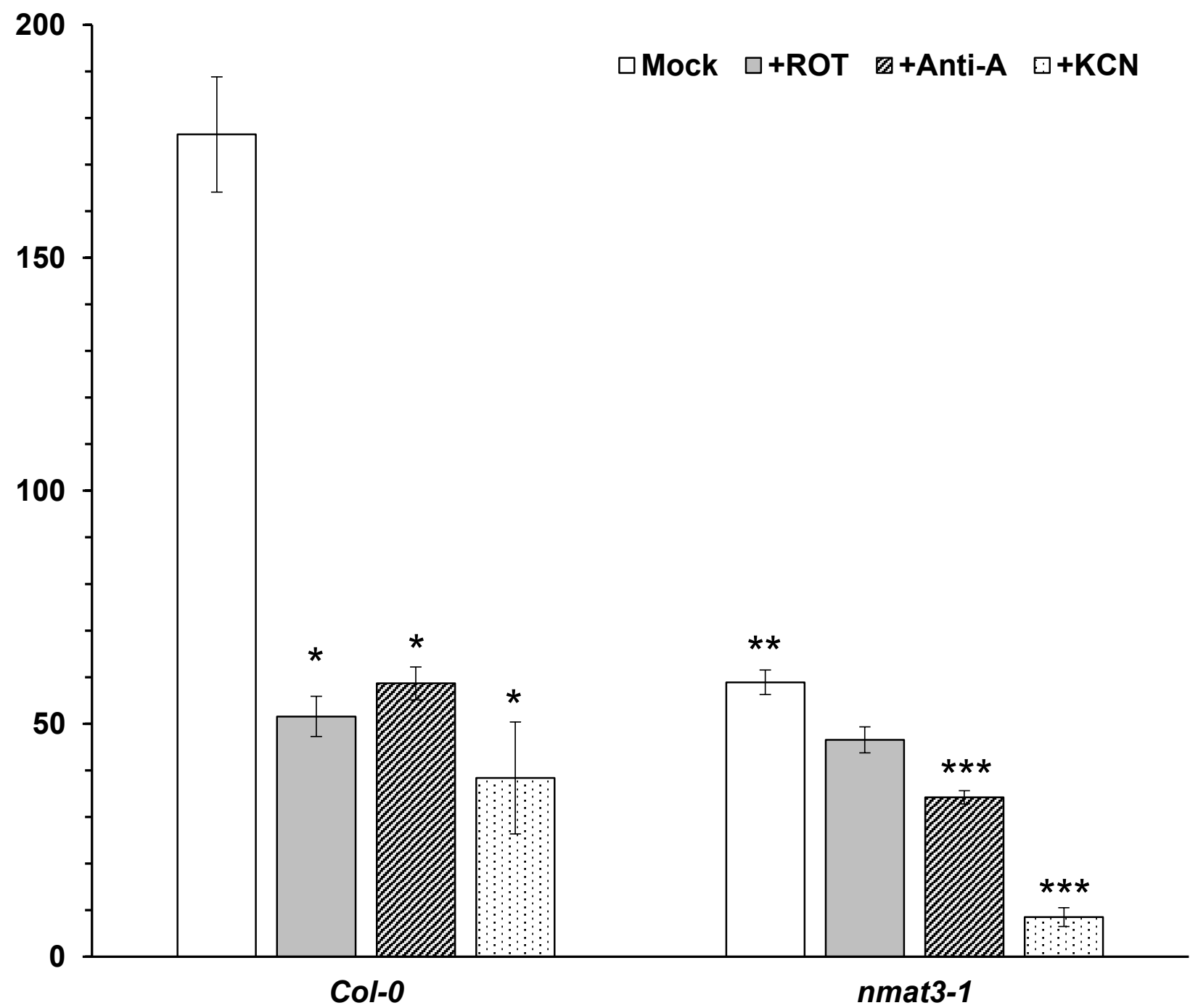

\begin{tabular}{|c|c|c|}
\hline & Col-0 & nmat3-1 \\
\hline $\begin{array}{c}\text { Mock } \\
\text { (mutant to WT ratio) }\end{array}$ & $100.00 \pm 7.00$ & $33.38 \pm 1.48$ \\
\hline $\begin{array}{c}\text { +ROT } \\
\text { (\% of inhibition) }\end{array}$ & $70.79 \pm 5.92$ & $20.99 \pm 6.00$ \\
\hline $\begin{array}{c}\text { +Anti-A } \\
\text { (\% of inhibition) }\end{array}$ & $66.76 \pm 6.03$ & $41.93 \pm 4.19$ \\
\hline $\begin{array}{c}\text { +KCN } \\
\text { (\% of inhibition) }\end{array}$ & $78.25 \pm 31.32$ & $85.57 \pm 23.82$ \\
\hline
\end{tabular}

\section{Figure 8}


nad1.1

(Atmg01275)

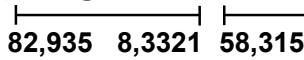

nad1.2

(ATMG01120)

58,397

82,935

(Atmg00516)

83,321

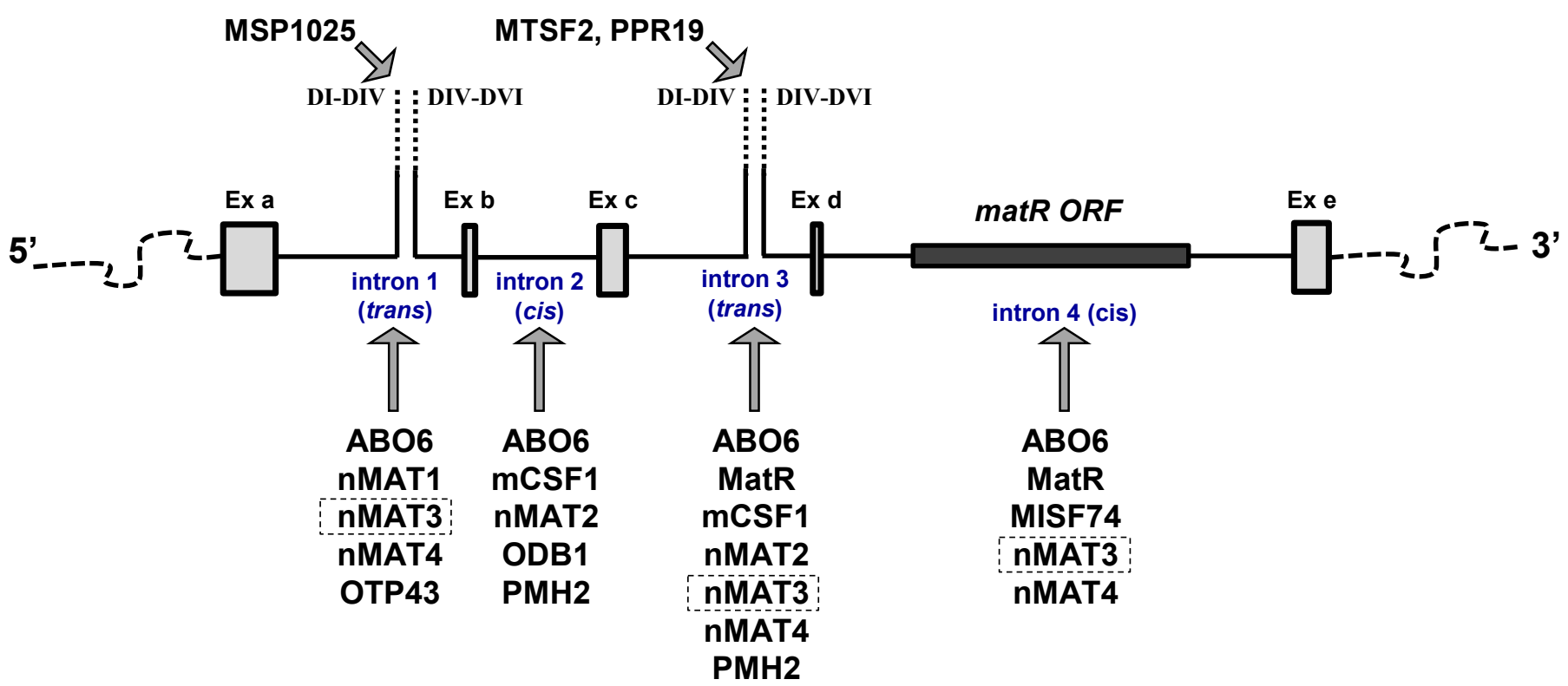

Figure 9 